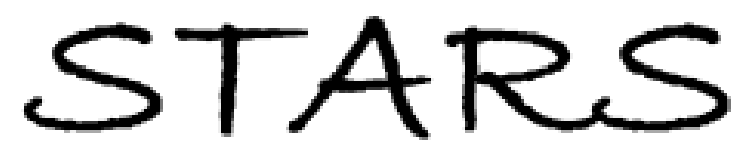

University of Central Florida

STARS

$1-1-2011$

\title{
Unified description of the dc conductivity of monolayer and bilayer graphene at finite densities based on resonant scatterers
}

Aires Ferreira

J. Viana-Gomes

Johan Nilsson

E. R. Mucciolo

University of Central Florida

N. M. R. Peres

See next page for additional authors

Find similar works at: https://stars.library.ucf.edu/facultybib2010

University of Central Florida Libraries http://library.ucf.edu

This Article is brought to you for free and open access by the Faculty Bibliography at STARS. It has been accepted for inclusion in Faculty Bibliography 2010 s by an authorized administrator of STARS. For more information, please contactSTARS@ucf.edu.

\section{Recommended Citation}

Ferreira, Aires; Viana-Gomes, J.; Nilsson, Johan; Mucciolo, E. R.; Peres, N. M. R.; and Neto, A. H. Castro, "Unified description of the dc conductivity of monolayer and bilayer graphene at finite densities based on resonant scatterers" (2011). Faculty Bibliography 2010s. 1289.

https://stars.library.ucf.edu/facultybib2010/1289

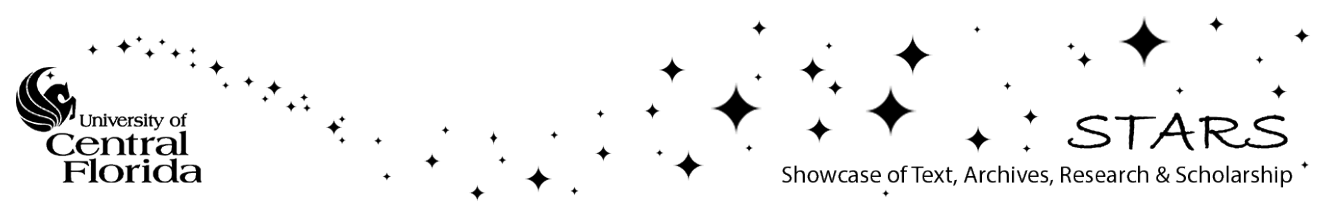


Authors

Aires Ferreira, J. Viana-Gomes, Johan Nilsson, E. R. Mucciolo, N. M. R. Peres, and A. H. Castro Neto 


\title{
Unified description of the dc conductivity of monolayer and bilayer graphene at finite densities based on resonant scatterers
}

\author{
Aires Ferreira, ${ }^{1}$ J. Viana-Gomes, ${ }^{1}$ Johan Nilsson, ${ }^{2}$ E. R. Mucciolo, ${ }^{3}$ N. M. R. Peres,,${ }^{1}{ }^{*}$ and A. H. Castro Neto ${ }^{4}$ \\ ${ }^{1}$ Department of Physics and Center of Physics, University of Minho, P-4710-057, Braga, Portugal \\ ${ }^{2}$ Department of Physics, University of Gothenburg, S-412 96 Gothenburg, Sweden \\ ${ }^{3}$ Department of Physics, University of Central Florida, Orlando, Florida 32816, USA \\ ${ }^{4}$ Department of Physics, Boston University, 590 Commonwealth Avenue, Boston, Massachusetts 02215, USA
}

(Received 25 October 2010; revised manuscript received 18 January 2011; published 4 April 2011)

\begin{abstract}
We show that a coherent picture of the dc conductivity of monolayer and bilayer graphene at finite electronic densities emerges upon considering that strong short-range potentials are the main source of scattering in these two systems. The origin of the strong short-range potentials may lie in adsorbed hydrocarbons at the surface of graphene. The equivalence among results based on the partial-wave description of scattering, the Lippmann-Schwinger equation, and the $T$-matrix approach is established. Scattering due to resonant impurities close to the neutrality point is investigated via a numerical computation of the Kubo formula using a kernel polynomial method. We find that relevant adsorbate species originate impurity bands in monolayer and bilayer graphene close to the Dirac point. In the midgap region, a plateau of minimum conductivity of about $e^{2} / h$ (per layer) is induced by the resonant disorder. In bilayer graphene, a large adsorbate concentration can develop an energy gap between midgap and high-energy states. As a consequence, the conductivity plateau is supressed near the edges and a "conductivity gap" takes place. Finally, a scattering formalism for electrons in biased bilayer graphene, taking into account the degeneracy of the spectrum, is developed and the dc conductivity of that system is studied.
\end{abstract}

DOI: $10.1103 /$ PhysRevB.83.165402

PACS number(s): 81.05.ue, 72.80.Vp, 78.67.Wj

\section{INTRODUCTION}

In his famous book, ${ }^{1}$ Peierls noted that in three dimensions the first Born approximation (FBA) cannot be used to deal with short-range potentials in general, even when the potential is not too strong. The reason lies in the fact that the FBA overestimates the value of the scattering cross section and modifies the energy dependence of the latter relative to the exact result. The fundamental reason why this effect takes place has its roots in the modification of the wave function within the region where the potential is finite. There, even for moderate potentials, the wave function is strongly deformed relative to the plane wave used in the FBA.

Since his main concern was nuclear physics, Peierls did not address the validity of the FBA in systems of reduced dimensions. Contrary to nuclear physics, some condensed matter systems impose dimensional constraints on the electronic motion-a direct consequence of the lattice structure of the given solid. Electrons moving in graphene face the most dramatic dimensional constraint, being forced to move along a strictly two-dimensional plane formed by a honeycomb lattice of carbon atoms. ${ }^{2-5}$ In bilayer graphene, electrons are also confined to move in two dimensions. Since bilayer systems are a stacking of two graphene sheets, the electrons may, additionally, hop between the layers.

Scattering cross sections in condensed matter physics are of ultimate importance for the interpretation of dc transport in solids, especially concerning the effect of localized impurities. These can be described by either short-range or long-range potentials. Following Peierls, ${ }^{1}$ the correct interpretation of the conductivity of a metal at low temperatures may require a description of electronic scattering by impurities beyond the FBA: this is particularly true if the impurities give rise to strong short-range potentials.
In systems such as monolayer and bilayer graphene, where the electronic density can be tuned between 0 and $\sim 10^{14} \mathrm{~cm}^{-2}$, Ref. 6 computing the correct dependence of the cross section on the Fermi energy is a crucial ingredient for a meaningful interpretation of the data. Since the early days of graphene physics, ${ }^{2,3}$ it has become clear that the conductivity of monolayer graphene shows a slightly sublinear dependence on electronic density. In contrast, the conductivity of bilayer graphene shows, consistently, a robust linear dependence on the backgate potential. Both monolayer and bilayer graphenebased field-effect devices use sheets from flakes produced in exactly the same manner, that is, via exfoliation of graphite. (More recently, graphene has been isolated via epitaxial growth on $\mathrm{SiC}^{7}$ and chemical vapor depositions on metal surfaces. ${ }^{8,9}$ ) It is now believed that the main sources of electronic scattering in exfoliated graphene are introduced during the device fabrication process.

The sources of disorder in graphene can vary. They can be due to adsorbed chemical species, such as hydrogen atoms or hydrocarbon molecules, random strain, ${ }^{11}$ rippling ${ }^{12,13}$ and scrolling, ${ }^{14}$ and electrostatic random potentials at the surface of the silicon oxide substrate caused by charged impurities. ${ }^{15-18}$ (Chemically synthesized graphene displays alternative scattering mechanisms. ${ }^{10}$ )

It is widely accepted that the strong carrier density fluctuations (electron-hole puddles) observed close to the neutrality or Dirac point are due to localized subsurface charged impurities. ${ }^{15,19}$ Whether charged impurities are also the limiting source of scattering in doped graphene (i.e., away from the neutrality point) remains unclear. In addition to charged scatterers, resonant scattering due to adsorbed hydrocarbons ${ }^{20}$ is currently ascending in the list of candidates limiting the electronic mobility in graphene. ${ }^{21-24}$ As we show in Sec. III A, adsorbed hydrocarbons can effectively act as 
strong short-range scatterers. Strong, short-range, resonant scatterers can be mimicked by vacancies in a lattice model. ${ }^{25-27}$ In magneto-optical transport studies of graphene, short-range scattering seems essential to explain the width of the cyclotronpeak at high magnetic field. ${ }^{25-28}$

Since the sources of scattering are likely introduced during the fabrication process, they must be the same for both monolayer and bilayer graphene. Therefore, a consistent theoretical description of the conductivity of graphene, at low temperatures and finite electronic densities, must be able to describe the experimental curves of both monolayer and bilayer graphene by invoking the same source of scattering. In this paper, we show that such a consistent theoretical description can be achieved by considering strong short-range potentials whose origin may lie in adsorbed chemical species at the surface of the material. Instrumental to our description is the critical analysis developed by Peierls: Calculation of the exact scattering cross sections is essential for a correct interpretation of the experimental data.

Before studying the dc conductivity for both monolayer and bilayer graphene at finite electronic densities, a task we defer to Sec. III, we first survey the scattering theory for electrons in these systems in Sec. II. This first step is essential for comprehension of the remaining sections.

In Sec. III, we show, using a simple and intuitive model, that the effect of adsorbed chemical species on graphene is equivalent to that of very strong on-site short-range potentials - the so-called resonant scatterers. Here, we use lattice-based numerical calculations of the density of states to show in some detail how this class of impurities affects the electronic structure of monolayer and bilayer graphene. Using a continuous formulation, we also show that the semiclassical dc conductivity of both monolayer and bilayer graphene at finite densities can be easily calculated using the intuitive approach to scattering given the partial-wave analysis. We apply the developed formalism to resonant scatterers and show that this type of short-range disorder accounts well for experimental data.

Further, we demonstrate the need for the computation of exact electronic scattering amplitudes when applying the Boltzmann approach to strong short-range potentials, an issue overlooked in the literature that we re-examine here. The validity of the semiclassical results at finite electronic densities and low impurity densities is established via a $T$-matrix calculation of the Kubo dc conductivity. Finally, by means of a numerical calculation based on the kernel polynomial method (KPM), we illustrate the breakdown of the semiclassical picture for electronic densities close to the neutrality point. These simulations explore the limit of finite impurity density, thus fully taking into account interference effects neglected in the Boltzmann approach.

In Sec. IV, we adapt the formalism of Secs. II and III to describe scattering when a perpendicular electric field is applied to bilayer graphene. Conclusions are drawn in Sec. V. Several technical aspects of our results are given in the Appendix.

We note that transport in monolayer and bilayer graphene was addressed by some of us in an ealier publication. ${ }^{55}$ However, it is important to remark that in the present work our goal is to provide a unified description of transport in both systems based on the same scattering mechanism. Also, it is shown that the transport properties of the bilayer graphene can be understood in a much simpler, intuitive, and transparent way using the standard scattering formalism of partial waves. In this regard, our present work is complementary to the study developed in Ref. 55. That is, the present work closes the circle of showing that for both graphene and its bilayer, a coherent and unified description of dc transport in these systems can be described by one and the same formalism, be it the more formal and mathematically demanding one of the transfer matrix or the intuitive and simple one of partial waves.

\section{PARTIAL-WAVE ANALYSIS IN GRAPHENE}

As discussed in Sec. I, calculation of the dc conductivity of a metal requires computing the transport cross section as accurately as possible. A well-established approach is based on the computation of the phase shifts induced in the scattered electron wave function by the scattering potential. If the phase shifts are known exactly, so is the cross section. Below, we set the notation and introduce the central quantities needed in this work by giving a concise presentation of the phaseshift approach to scattering in the context of graphene and its bilayer. ${ }^{29-32}$ These results are later used in Sec. III. Also, and to the best of our knowledge, the scattering theory for electrons in a biased graphene bilayer has not been developed so far in the literature, and therefore it is presented in Sec. IV.

Scattering theory states that the large-distance wave function of a particle in the presence of a scattering potential (with cylindrical symmetry) must have the form (in two dimensions)

$$
\psi(\mathbf{r}) \simeq e^{i k_{i} x}+f(\theta) \frac{e^{i k_{f} r}}{\sqrt{r}},
$$

where $\mathbf{k}_{i}=\left(k_{i}, 0\right)$ and $\mathbf{k}_{f}=k_{f}(\cos \theta, \sin \theta)$ are the momentum of the incoming and scattered waves, respectively; clearly, for elastic scattering, we have $k_{i}=k_{f}=k$. The scattering amplitude $f(\theta)$ can be written in terms of the phase shifts $\delta_{m}$ associated with the partial-wave expansion of the scattered wave function in the basis of angular momentum states. In Eq. (1), the first term represents the incoming particle, with the incoming momentum oriented along the $x$ axis, and the second one represents the cylindrical scattered wave function.

As it stands, Eq. (1) holds for the two-dimensional Schrödinger equation. ${ }^{33}$ However, for both monolayer and bilayer graphene, the long-distance behavior of the wave function differs slightly, but significantly, from Eq. (1).

\section{A. Electronic scattering in graphene}

For graphene, the motion of the electrons in the $\pi$ orbitals is, at low energies, described by the two-dimensional massless Dirac Hamiltonian, reading ${ }^{4}$

$$
H_{K}=v_{F} \boldsymbol{\sigma} \cdot \mathbf{p},
$$

where the Fermi velocity is defined as $v_{F}=3 t a_{0} /(2 \hbar), t$ is the hopping integral between the $p_{z}$ orbitals of two adjacent carbon atoms, and $a_{0} \approx 1.4 \AA$ is the carbon-carbon distance in graphene (see Fig. 1). The vector $\sigma$ is written in terms of Pauli's matrices as $\boldsymbol{\sigma}=\left(\sigma_{x}, \sigma_{y}\right)$, and $\mathbf{p}$ is the momentum operator. The vector $\boldsymbol{K}$ denotes one of the two (inequivalent) 

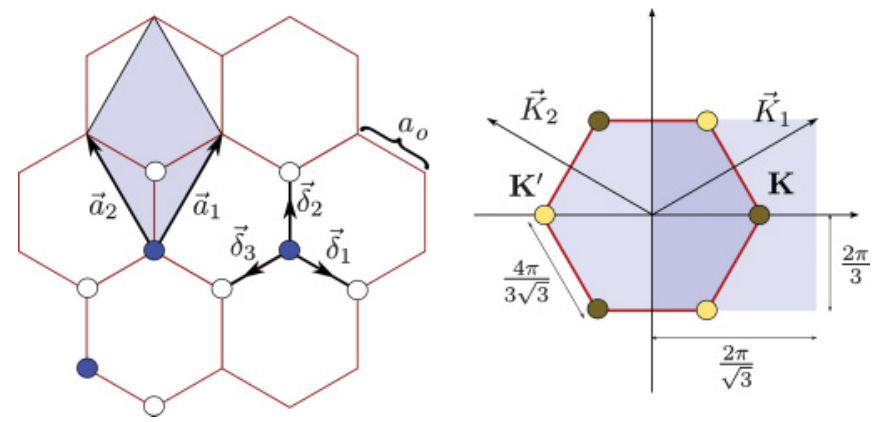

FIG. 1. (Color online) Lattice structure and Brillouin zone of monolayer graphene. Left: Hexagonal lattice of graphene, with the next nearest neighbor, $\boldsymbol{\delta}_{i}$, and the primitive, $\boldsymbol{a}_{i}$, vectors depicted. The area of the primitive cell is $A_{c}=3 \sqrt{3} a_{0}^{2} / 2 \simeq 5.1 \AA^{2}$, and $a_{0} \simeq 1.4 \AA$. Right: Brillouin zone of graphene, with the Dirac points $\boldsymbol{K}$ and $\boldsymbol{K}^{\prime}$ indicated. Close to these points, the dispersion of graphene is conical and the density of states is proportional to the absolute value of the energy.

edge points of the Brillouin zone, also called Dirac points or valleys. Because neutral graphene is half-filled (i.e., the $\pi$ orbitals contain one electron), these two points control the low-energy physics. Depending on the nature of disorder and the Fermi energy, coupling between momentum states from different valleys can take place. Intervalley scattering is known to induce weak localization corrections to the conductivity and, ultimately, fully localize states in the thermodynamic limit at zero temperature. . $^{6,73,74}$

In what follows, we assume that the two Dirac points, $\boldsymbol{K}$ and $\boldsymbol{K}^{\prime}$, can be treated independently. This procedure is justified because intervalley scattering (known to occur for short-range scatterers) manifests itself primarily in the coherent regime, through backscattering interference. For low concentrations of scattering centers, finite sample size, and finite temperatures (the typical experimental situation), coupling between the Dirac points can be neglected when considering high enough electronic densities. Hence, with the exception of the lattice calculations (Secs. IIIE and IIIG), we neglect intervalley scattering in the continuous model calculations and introduce the valley degeneracy index, $g_{v}=2$. In Cartesian coordinates, the eigenstate of the Hamiltonian in Eq. (2) has the explicit form

$$
\Psi_{ \pm}(\mathbf{r})=\frac{1}{\sqrt{2 A}}\left[1 \pm e^{i \theta_{\mathbf{k}}}\right] e^{i \mathbf{k} \cdot \mathbf{r}},
$$

with $\theta_{\mathbf{k}}=\arctan \left(k_{y} / k_{x}\right)$ and $A$ denoting the total area of the system. The energy eigenvalues corresponding to the eigenfunction in Eq. (3) are $E= \pm v_{F} \hbar k$. From the latter follows the density of states per spin and per unit cell, $\rho(E)=2|E| /\left(\pi \sqrt{3} t^{2}\right)$, where the contribution from the two valleys has been taken into account. The probability density current reads ${ }^{34}$

$$
\boldsymbol{J}=v_{F} \Psi_{ \pm}^{\dagger} \boldsymbol{\sigma} \Psi_{ \pm}
$$

For the study of scattering, it is more convenient to recast the Hamiltonian in Eq. (2) in cylindrical coordinates $r$ and $\theta$ as

$$
H_{K}=-i v_{F} \hbar\left[\begin{array}{cc}
0 & \hat{L}_{-} \\
\hat{L}_{+} & 0
\end{array}\right] \text {, }
$$

where the operators $\hat{L}_{ \pm}=e^{ \pm i \theta}\left(\partial_{r} \pm i r^{-1} \partial_{\theta}\right)$ act as rising/lowering operators, according to the following result:

$$
\hat{L}_{ \pm}\left[C_{m}(k r) e^{i \theta m}\right]=\mp k C_{m \pm 1}(k r) e^{i \theta(m \pm 1)} .
$$

In Eq. (6), the function $C_{m}(k r)$ stands for $J_{m}(k r)$ and $Y_{m}(k r)$, the first-kind and second-kind Bessel functions, respectively, and for the Hankel functions of the first kind $H_{m}^{(1)}$ and second kind $H_{m}^{(2)}$. For the modified Bessel function $K_{m}(k r)$ we have

$$
\hat{L}_{ \pm}\left[K_{m}(k r) e^{i \theta m}\right]=-k K_{m \pm 1}(k r) e^{i \theta(m \pm 1)} .
$$

In cylindric coordinates, the radial probability density current reads

$$
J_{r}=v_{F} \Psi_{ \pm}^{\dagger} \sigma_{r} \Psi_{ \pm},
$$

where $\sigma_{r}$ is defined as

$$
\sigma_{r}=\left[\begin{array}{cc}
0 & e^{-i \theta} \\
e^{i \theta} & 0
\end{array}\right] .
$$

The tangential component of the probability density current reads $J_{\theta}=v_{F} \Psi_{ \pm}^{\dagger} \sigma_{\theta} \Psi_{ \pm}$, with $\sigma_{\theta}=\sigma_{r} \operatorname{diag}(i,-i)$, and where $\operatorname{diag}(i,-i)$ represents a diagonal matrix. Let us now derive, for massless Dirac electrons in two dimensions, the equivalent of the asymptotic wave function in Eq. (1). To that end, we note that a state having the form

$$
\Psi_{m}(r, \theta)=\frac{1}{\sqrt{2 A}}\left[J_{m}(k r) \pm i e^{i \theta} J_{m+1}(k r)\right] e^{i m \theta}
$$

is also an eigenstate of the Hamiltonian in Eq. (5). We start by assuming that the asymptotic (large $r$ ) behavior of the wave function in the angular momentum channel $m$ has the form (from here on, we consider only $E>0$ )

$$
\Psi_{m}(r, \theta) \simeq \sqrt{\frac{1}{\pi A k r}}\left[\begin{array}{c}
\cos \left(k r-\lambda_{m}+\delta_{m}\right) \\
i e^{i \theta} \sin \left(k r-\lambda_{m}+\delta_{m}\right)
\end{array}\right] e^{i\left(m \theta+\delta_{m}\right)},
$$

an ansatz inspired by the fact that the Dirac equation for graphene is a set of two coupled first-order differential equations and in the asymptotic limit of the Bessel functions at large $r:{ }^{35}$

$$
\begin{aligned}
& J_{m}(x)=\sqrt{\frac{2}{\pi x}} \cos \left(x-\lambda_{m}\right), \\
& Y_{m}(x)=\sqrt{\frac{2}{\pi x}} \sin \left(x-\lambda_{m}\right),
\end{aligned}
$$

with $\lambda_{m}=m \pi / 2+\pi / 4$. Using Eq. (11), we write the total wave function as an expansion in partial waves, reading

$$
\Psi(r, \theta)=\sum_{m=-\infty}^{\infty} i^{m} \Psi_{m}(r, \theta) .
$$

Exploiting of the relation

$$
e^{i k r \cos \theta}=\sum_{m=-\infty}^{\infty} i^{m} e^{i m \theta} J_{m}(k r),
$$

we obtain

$$
\Psi(\mathbf{r}) \simeq \frac{1}{\sqrt{2 A}}\left[\begin{array}{l}
1 \\
1
\end{array}\right] e^{i k x}+\frac{1}{\sqrt{2 A}}\left[\begin{array}{c}
1 \\
e^{i \theta}
\end{array}\right] f(\theta) \frac{e^{i k r}}{\sqrt{r}},
$$


with the scattering amplitude reading

$$
f(\theta)=\sqrt{\frac{2 i}{\pi k}} \sum_{m=-\infty}^{\infty} e^{i m \theta} e^{i \delta_{m}} \sin \delta_{m} .
$$

It is a simple exercise to show that the first term in Eq. (16) corresponds to a flux $J_{x}=v_{F} / A$ (and $J_{y}=0$ ), whereas the second term corresponds to a radial flux $J_{r}=v_{F}|f(\theta)|^{2} /(r A)$ (and $J_{\theta}=0$ ). Thus, according to the usual definition of the differential cross section $\sigma(\theta)$, it follows that

$$
\sigma(\theta)=|f(\theta)|^{2}
$$

Before we turn to scattering in bilayer graphene, it will be useful, for later use, to introduce other asymptotic forms of the Bessel functions $J_{m}(x), Y_{m}(x)$, and $K_{m}(x)$, in addition to those already given in Eqs. (12) and (13). For large $x$, we have ${ }^{35}$

$$
K_{m}(x)=\sqrt{\frac{\pi}{2 x}} e^{-x}
$$

For $x \ll 1$, the asymptotic forms read ${ }^{35}$

$$
\begin{gathered}
J_{m}(x)=(x / 2)^{m} \Gamma^{-1}(m+1), \\
Y_{0}(x)=2 \pi^{-1} \ln x, \\
Y_{m}(x)=-\pi^{-1} \Gamma(m)(x / 2)^{-m}, \quad m=1,2, \ldots,
\end{gathered}
$$

and

$$
\begin{gathered}
K_{0}(x)=-\ln x, \\
K_{m}(x)=2^{-1} \Gamma(m)(x / 2)^{-m}, \quad m=1,2, \ldots,
\end{gathered}
$$

where $\Gamma(x)$ is the $\Gamma$ function. We now consider scattering in bilayer graphene.

\section{B. Electronic scattering in bilayer graphene}

Bilayer graphene has four atoms per unit cell, with the two honeycomb sheets arranged according to a Bernal stacking, as shown in Fig. 2. Two of the atoms belonging to each of the layers are on top of each other (atoms $A_{1}$ and $B_{2}$ in Fig. 2), allowing for interlayer hopping. This process is represented by a hopping parameter, $t_{\perp} \approx 0.5 \mathrm{eV} .^{36,37}$ The other two carbon atoms, labeled $A_{2}$ and $B_{1}$ in Fig. 2, are not coupled to the carbon atoms of the other layer, in accordance with the assumptions of the minimal model for electronic motion in bilayer graphene.

The band structure of bilayer graphene has four bands, but the low-energy physics $\left(|E| \ll t_{\perp}\right)$ can be described by an effective model of only two bands, ${ }^{36-38}$ where the atoms linked by $t_{\perp}$ are projected out since they describe high-energy bands: the dimmer of atoms $A_{1}$ and $B_{2}$, linked by $t_{\perp}$, form a two-level system with energy levels $\pm t_{\perp}$. Additionally, the atoms in the two sheets can be made nonequivalent by applying an electric field perpendicular to the sheets, in this way inducing a gap in the spectrum (the electrostatic potential difference between the two layers is $2 V) .{ }^{36-39}$

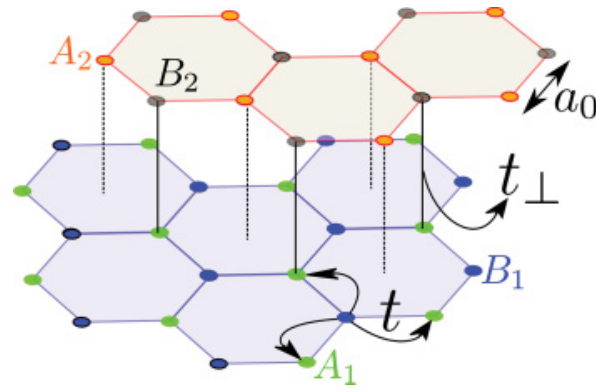

FIG. 2. (Color online) Lattice structure of bilayer graphene. The atoms labeled $A_{1}$ and $B_{1}$ lie on the bottom graphene layer, whereas atoms $A_{2}$ and $B_{2}$ are in the top layer. Electrons can hop between layers via a perpendicular hopping parameter $t_{\perp}$ between carbon atom $A_{1}$ and carbon atom $B_{2}$ (connected by solid lines). The Brillouin zone of bilayer graphene is the same as that of monolayer graphene (see Fig. 1).

The derivation of the effective Hamiltonian is straightforward. We write the full Hamiltonian as

$$
H=\left[\begin{array}{cccc}
V & 0 & 0 & \hat{\pi} \\
0 & -V & \hat{\pi}^{\dagger} & 0 \\
0 & \hat{\pi} & -V & -t_{\perp} \\
\hat{\pi}^{\dagger} & 0 & -t_{\perp} & V
\end{array}\right] \equiv\left[\begin{array}{cc}
H_{\mathrm{L}} & H_{\mathrm{LH}} \\
H_{\mathrm{LH}}^{\dagger} & H_{\mathrm{H}}
\end{array}\right],
$$

where the columns in the Hamiltonian are labeled by the four atoms in the unit cell. In ascending order, this labeling is $B_{1}$, $A_{2}, B_{2}$, and $A_{1}$. The operator $\hat{\pi}$ is defined as $\hat{\pi} \equiv v_{F}\left(\hat{p}_{x}+\right.$ $i \hat{p}_{y}$ ). The eigenproblem $H|\psi\rangle=E|\psi\rangle$ can be written as

$$
\left[\begin{array}{cc}
H_{\mathrm{L}} & H_{\mathrm{LH}} \\
H_{\mathrm{LH}}^{\dagger} & H_{\mathrm{H}}
\end{array}\right]\left[\begin{array}{c}
|\varphi\rangle \\
|\chi\rangle
\end{array}\right]=E\left[\begin{array}{l}
|\varphi\rangle \\
|\chi\rangle
\end{array}\right] \text {. }
$$

It follows from Eq. (26) that

$$
H_{\mathrm{L}}|\varphi\rangle+H_{\mathrm{LH}}\left(E-H_{\mathrm{H}}\right)^{-1} H_{\mathrm{LH}}^{\dagger}|\varphi\rangle=E|\varphi\rangle,
$$

and considering that $t_{\perp} \gg(V,|E|)$, we have $H_{\mathrm{BL}}|\varphi\rangle=E|\varphi\rangle$, with $^{38}$

$$
H_{\mathrm{BL}}=V \sigma_{z}-\frac{V}{t_{\perp}^{2}}\left[\begin{array}{cc}
\hat{\pi} \hat{\pi}^{\dagger} & 0 \\
0 & -\hat{\pi}^{\dagger} \hat{\pi}
\end{array}\right]+\frac{1}{t_{\perp}}\left[\begin{array}{cc}
0 & (\hat{\pi})^{2} \\
\left(\hat{\pi}^{\dagger}\right)^{2} & 0
\end{array}\right] .
$$

To keep things simple, in what follows we consider the case $V=0$; later we discuss the case $V \neq 0$. In cylindric coordinates, the Hamiltonian, Eq. (28), is written as

$$
H_{\mathrm{BL}}=-\frac{v_{F}^{2} \hbar^{2}}{t_{\perp}}\left[\begin{array}{cc}
0 & \hat{L}_{-}^{2} \\
\hat{L}_{+}^{2} & 0
\end{array}\right],
$$

and the eigenfunctions (regular at the origin) can be written as

$$
\Psi_{m}(r, \theta)=\frac{1}{\sqrt{2 A}}\left[J_{m}(k r) \mp e^{2 i \theta} J_{m+2}(k r)\right] e^{i m \theta},
$$

to which the eigenvalues $E= \pm v_{F}^{2} \hbar^{2} k^{2} / t_{\perp}$ correspond. From the latter result follows the density of states per spin and per unit cell, $\rho(E)=t_{\perp} /\left(\pi \sqrt{3} t^{2}\right)$, where we have included a factor of 2 coming from the valley degeneracy. ${ }^{71}$

It is important to stress two differences between the Hamiltonians in Eqs. (2) and (29) regarding boundary conditions and the nature of the scattering states. To be concrete, let us assume that the electron is subjected to a potential well of the form 
$V(r)=V_{0} \theta(R-r)$. In the case of the Dirac Hamiltonian, the boundary conditions at $r=R$ imply continuity of the two components of the spinors, whereas for the bilayer Hamiltonian we have to impose continuity of both the components of the spinors and their first derivative. The second aspect is related to the fact that elastic scattering conserves energy. Thus, since in bilayer graphene we have $E= \pm v_{F}^{2} \hbar^{2} k^{2} / t_{\perp}$, and keeping the energy constant, say $E>0$, as in any scattering process, there are two admissible solutions: a real solution, $k=$ $\sqrt{t_{\perp} E} /\left(v_{F} \hbar\right)$, and a purely imaginary one, $k=i \sqrt{t_{\perp} E} /\left(v_{F} \hbar\right)$. Therefore, bilayer graphene supports evanescent modes at the interface $r=R$. This fact is essential to satisfy the boundary conditions obeyed by the wave function. ${ }^{40}$

As in the case of the Dirac Hamiltonian, we have to derive the form of the probability density current for electrons described by the Hamiltonian in Eq. (29). The usual procedure ${ }^{34}$ gives that any component $J_{\ell}$ of the current has the form

$$
J_{\ell}=2 \frac{v_{F}^{2} \hbar}{t_{\perp}} \operatorname{Im} \Psi^{\dagger} \hat{J}_{\ell} \Psi
$$

where for $\ell=x, y$ we have

$$
\hat{J}_{x}=\sigma_{x} \partial_{x}+\sigma_{y} \partial_{y}
$$

and

$$
\hat{J}_{y}=\sigma_{y} \partial_{x}-\sigma_{x} \partial_{y} .
$$

For the radial component, $\ell=r$, we have

$$
\hat{J}_{r}=\left[\begin{array}{cc}
0 & e^{-2 i \theta}\left(\partial_{r}+i r^{-1} \partial_{\theta}\right) \\
e^{2 i \theta}\left(\partial_{r}-i r^{-1} \partial_{\theta}\right) & 0
\end{array}\right],
$$

and for the tangential component, $\ell=\theta$, we have

$$
\hat{J}_{\theta}=\left[\begin{array}{cc}
0 & -i e^{-2 i \theta}\left(\partial_{r}-i r^{-1} \partial_{\theta}\right) \\
i e^{2 i \theta}\left(\partial_{r}+i r^{-1} \partial_{\theta}\right) & 0
\end{array}\right] .
$$

Taking into account that the Hamiltonian in Eq. (29) forms a set of two coupled second-order differential equations, we assume that the asymptotic (large- $r$ ) behavior of the wave function in the angular momentum channel $m$ has the form

$$
\Psi_{m}(r, \theta) \simeq \sqrt{\frac{1}{\pi A k r}}\left[\begin{array}{c}
\cos \left(k r-\lambda_{m}+\delta_{m}\right) \\
e^{2 i \theta} \cos \left(k r-\lambda_{m}+\delta_{m}\right)
\end{array}\right] e^{i\left(m \theta+\delta_{m}\right)} .
$$

Following the same procedure used to derive Eq. (16), we can show that the large- $r$ behavior of the total electronic wave function in graphene bilayer in the presence of a potential has the form

$$
\Psi(\mathbf{r}) \simeq \frac{1}{\sqrt{2 A}}\left(\begin{array}{l}
1 \\
1
\end{array}\right) e^{i k x}+\frac{1}{\sqrt{2 A}}\left(\begin{array}{c}
1 \\
e^{2 i \theta}
\end{array}\right) f(\theta) \frac{e^{i k r}}{\sqrt{r}} .
$$

Using Eq. (31), we can easily conclude that the first term in Eq. (37) corresponds to a flux $J_{x}=2 v_{F}^{2} \hbar k /\left(A t_{\perp}\right) \equiv v / A$, where $v$ is the velocity of the particle, and that the second term corresponds to a radial flux of the form $J_{r}=$ $2 v_{F}^{2} \hbar k|f(\theta)|^{2} /\left(r A t_{\perp}\right) \equiv v|f(\theta)|^{2} /(A r)$, with $f(\theta)$ still given by Eq. (17). As before, it follows that the differential cross section is given by Eq. (18).

In Sec. III, we apply this formalism to the case of a potential well described by the potential $V(r)=V_{0} \theta(R-r)$ in the strongly interacting regime $V_{0} \gg t$. We will see that the results are insensitive to the particular form adopted for $V(r)$ as long it corresponds to a strong short-range potential.

\section{THE DC CONDUCTIVITY OF GRAPHENE AND ITS BILAYER}

As discussed in Sec. I, there is growing evidence that the limiting scattering mechanism of the electronic mobility in graphene is due to strong short-range potentials, likely to have originated from adsorbed hydrocarbons. These adsorbed atoms and/or molecules act as resonant scatterers, giving rise to midgap states. ${ }^{20,41-43}$

This section is most important: it clarifies why the statement that short-range scatterers in graphene give a dc conductivity independent of the gate voltage is erroneous. As noted in Sec. I, this misleading idea has its roots in the FBA, which fails blatantly in this problem, as we demonstrate in what follows.

\section{A. Adsorbed atoms in graphene as strong short-range scattering centers}

The resonant scattering mechanism is easy to seize by considering a simple model. Let us write the tight-binding Hamiltonian of the $\pi$ electrons in graphene as (spin index omitted)

$$
H=-t \sum_{n, \delta_{i}}\left|A, \boldsymbol{R}_{n}\right\rangle\left\langle\boldsymbol{R}_{n}+\boldsymbol{\delta}_{i}, B\right|+\text { H.c. },
$$

where $\left|A, \boldsymbol{R}_{n}\right\rangle$ represents the Wannier state at the unit cell $\boldsymbol{R}_{n}$; an equivalent definition holds for $\left|B, \boldsymbol{R}_{n}+\boldsymbol{\delta}_{i}\right\rangle$, where $\boldsymbol{\delta}_{i}$ is one of three nearest-neighbor vectors in the honeycomb lattice, as depicted in Fig. 1.

We now consider that an impurity is binding covalently to a carbon atom at site $\boldsymbol{R}_{n}=0$. This situation adds to the Hamiltonian in Eq. (38) a term of the form

$$
H_{\mathrm{rs}}=\left(V_{\mathrm{ad}}|\mathrm{ad}\rangle\langle A, 0|+\text { H.c. }\right)+\epsilon_{\mathrm{ad}}|\mathrm{ad}\rangle\langle\mathrm{ad}|,
$$

where $V_{\text {ad }}$ is the hybridization between the adatom (or a carbon atom of a hydrocarbon molecule) and a given carbon atom of graphene, $\epsilon_{\mathrm{ad}}$ is the relative (to graphene's carbon atoms) on-site energy of the electron in the adatom, and $|\mathrm{ad}\rangle$ is the ket representing the state of the electron in the adatom. Taking the wave function to be of the form

$$
\begin{aligned}
|\psi\rangle= & \sum_{n}\left[A\left(\boldsymbol{R}_{n}\right)\left|A, \boldsymbol{R}_{n}\right\rangle+B\left(\boldsymbol{R}_{n}+\boldsymbol{\delta}_{2}\right)\left|B, \boldsymbol{R}_{n}+\boldsymbol{\delta}_{2}\right\rangle\right] \\
& +C_{\mathrm{ad}}|\mathrm{ad}\rangle,
\end{aligned}
$$

the Schrödinger equation applied to the site $\boldsymbol{R}_{n}=0$ reads

$$
\begin{aligned}
E A(0)-V_{\mathrm{ad}} C_{\mathrm{ad}} & =-t\left[B\left(\boldsymbol{\delta}_{1}\right)+B\left(\boldsymbol{\delta}_{2}\right)+B\left(\boldsymbol{\delta}_{3}\right)\right], \\
\left(E-\epsilon_{\mathrm{ad}}\right) C_{\mathrm{ad}} & =V_{\mathrm{ad}} A(0) .
\end{aligned}
$$

Solving for $C_{\mathrm{ad}}$, we obtain

$$
-t\left[B\left(\boldsymbol{\delta}_{1}\right)+B\left(\boldsymbol{\delta}_{2}\right)+B\left(\boldsymbol{\delta}_{3}\right)\right]=\left(E-\frac{V_{\mathrm{ad}}^{2}}{E-\epsilon_{\mathrm{ad}}}\right) A(0) .
$$


The resonant effect is included in the last term in Eq. (42), which represents an effective local potential of strength

$$
V_{\text {eff }}=V_{\text {ad }}^{2} /\left(E-\epsilon_{\text {ad }}\right) .
$$

Quantum chemical calculations can determine the value of the parameters $\epsilon_{\text {ad }}$ and $V_{\text {ad }} .^{20,41,43}$ Typical values are $V_{\text {ad }} \sim$ $2 t \sim 5 \mathrm{eV}$ and $\epsilon_{\mathrm{ad}} \sim-0.2 \mathrm{eV},{ }^{20}$ leading to $V_{\text {eff }} \sim 100 \mathrm{eV}$ at half-filling $(E=0)$, a rather strong on-site potential. On the basis of this fact, it is natural to expect that adsorbates (i.e., resonant scatterers) and vacancies lead to similar effects on the electronic structure and transport properties. In monolayer graphene, vacancies are known to significantly alter the density of states at energies close to $\epsilon_{\mathrm{ad}}$. In particular, vacancies induce a large spectral transference from the Van Hove singularities to the neighborhood of the Dirac point. As a consequence, the density of states displays sharp peaks within the midgap region. ${ }^{26,44}$ This effect was first demonstrated in Ref. 26; recently, it has been shown that indeed adsorbates do originate similar behavior. ${ }^{20,72}$

Here we report similar results for bilayer graphene. To calculate the density of states, we employ the KPM (see Ref. 62 for a review). For the sake of simplicity, we have considered equal concentrations of adsorbates in both bottom and top layers. (The actual applicability of this choice depends on the laboratory conditions and specific experimental setup.) In what follows, we discuss the situation where the adatoms bind only to carbons with coordination number $z=3$ (i.e., those termed $A_{2}$ and $B_{1}$ in Fig. 2).

The effect of resonant impurities in the electronic structure of monolayer and bilayer graphene for different adsorbates concentrations, $n_{\mathrm{ad}}$, per carbon atom, is shown in Fig. 3. For illustration purposes, we present the results for a high defect concentration, $n_{\mathrm{ad}} \sim 1 \%$, so that the modification of the graphene electronic structure is visible to the eye in a wide energy window; later we will see that the estimated values for defect concentration, for typical experimental conditions, are actually far below these values (Secs. III C and III D).

In both graphene systems, the adatoms lead to well-defined peaks close to zero energy, the so-called midgap region. As mentioned above, such enhancement of the density of states is accompanied by a decrease in spectral weight near the Van Hove singularities, a situation reminiscent of vacancy-induced disorder. $^{26,44}$ The effective potential [Eq. (43)], despite being very strong, is bounded, explaining the slight electron-hole asymmetry near the Dirac point. The resonant peaks are centered at negative energies because $\epsilon_{\mathrm{ad}}<0$. Increasing the impurity concentration brings more spectral weight toward the midgap region. In bilayer graphene, though, a curious phenomenon takes place: when the impurity concentration is large enough, a gap opens separating midgap states, forming the impurity band, from high-energy states (see Fig. 3, bottom). Similar findings were reported in recent $a b$ initio calculations considering asymmetric doping of graphene. ${ }^{75}$

Figure 4 shows how the electronic structure changes when the restriction on the allowable carbon-impurity bonds is relaxed. When adsorbates bind to carbons in any sublattice in the bilayer, the density of states is almost indistinguishable from that of monolayer graphene (with the same impurity concentration). The latter is accurate for a large energy window around the Dirac point $(|\epsilon| \lesssim 0.5 t)$; for higher electronic
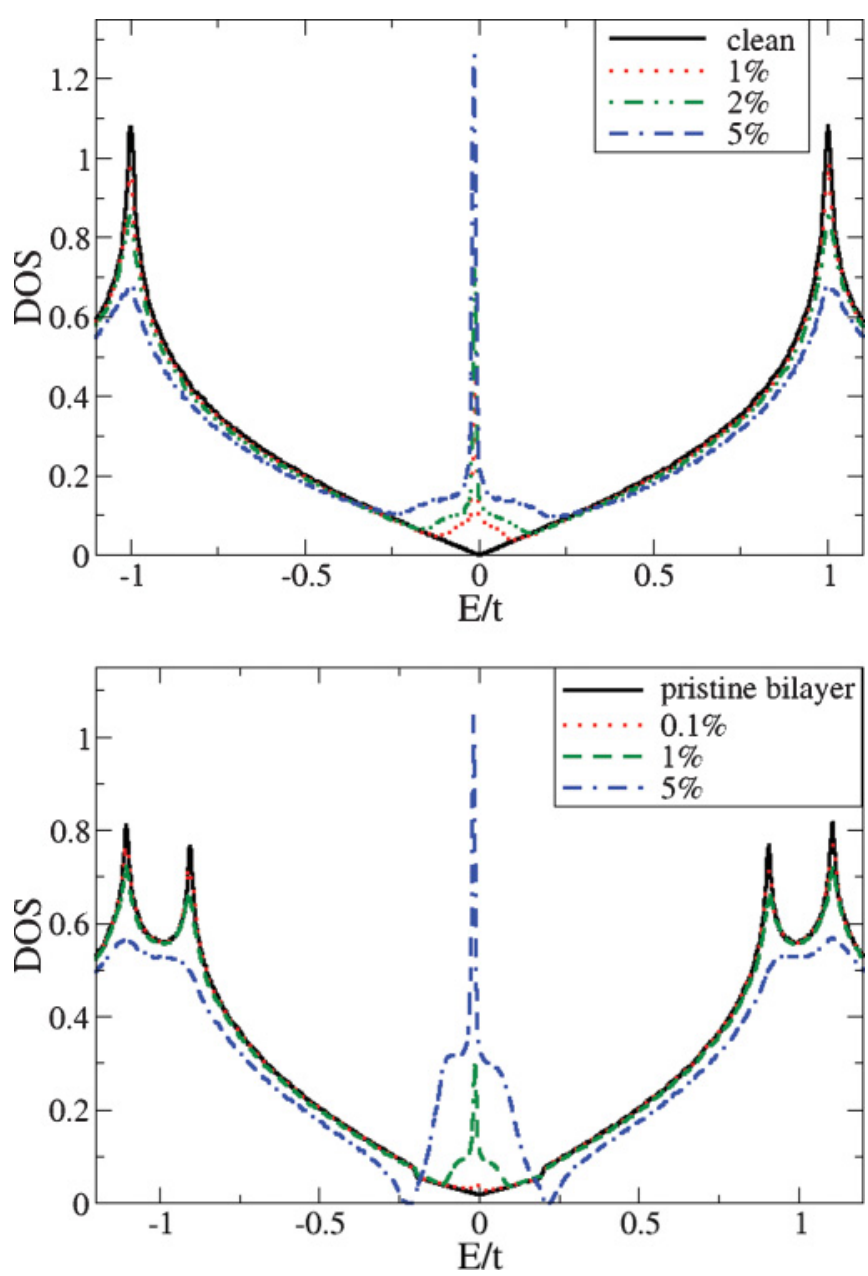

FIG. 3. (Color online) Effect of adatoms (resonant scatterers) on the density of states (DOS) of monolayer graphene (top) and bilayer graphene (bottom). Calculation of the DOS was carried out in honeycomb lattices with $N=1000 \times 1000$ carbon sites for different concentrations of adsorbed atoms (periodic boundary conditions and 10 realizations of disorder were taken). The tight-binding parameters read $V_{\text {ad }}=2 t, \epsilon_{\text {ad }}=-0.0625 t$, and $t_{\perp}=0.2 t$. The DOS discloses a dislocation of spectral weight toward the midgap region, a phenomenon first reported for vacancies in monolayer graphene in Ref. 26.

energies, the density of states becomes insensitive to the type of impurity-carbon bonds present in the bilayer samples. Roughly speaking, forming chemical bonds to every type of carbon decouples the layers, and hence dc-transport properties will be similar to those of a single layer of graphene (Sec. III G).

In light of the present results and previous reports for vacancies ${ }^{26,44}$ and resonant impurities in monolayer graphene, ${ }^{20,72}$ we are led to conclude that the formation of an impurity band in the midgap region is universal in graphene systems with typical adsorbed species. In Sec. III G, it will be shown that such an impurity band has a strong impact on the transport properties of undoped graphene.

Away from neutrality, the calculation of transport properties for the effective local potential model can be performed using the $T$-matrix approach. ${ }^{41,45,46}$ Its derivation for resonant scatterers is elementary. It is well known that the $T$ matrix for 


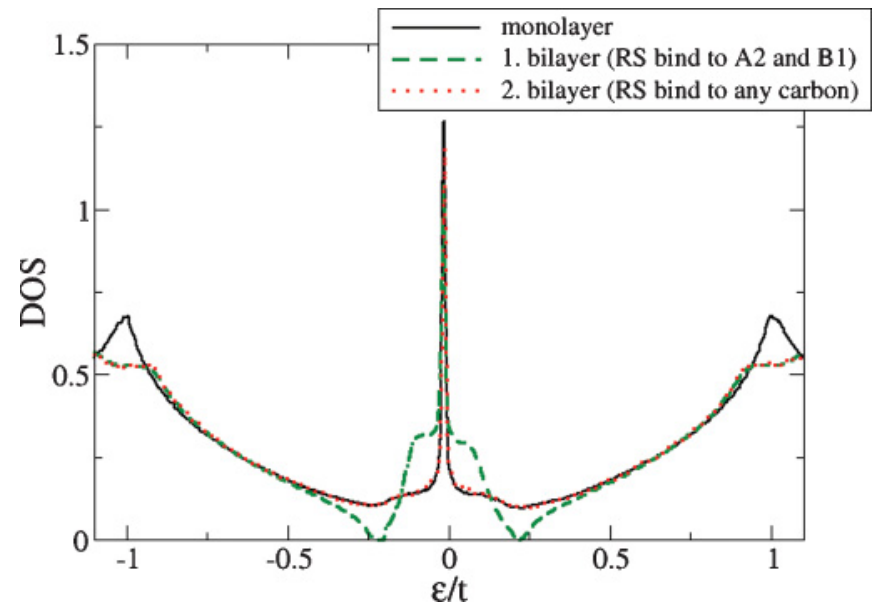

FIG. 4. (Color online) Density of states (DOS) for bilayer systems with 5\% resonant scatterers (RS) in the two scenarios described in the text, namely, (1) adsorbates binding only to carbons $A_{2}$ and $B_{1}$ and (2) adsorbates forming bonds with carbons in any sublattice. The first situation opens a gap between the impurity band and high-energy states. The DOS of monolayer graphene is shown for comparison; tight-binding parameters are given in the caption to Fig. 3.

a local potential of intensity $v_{0}$ reads ${ }^{25,47}$

$$
T(E)=v_{0}\left[1-v_{0} \bar{G}_{R}(E)\right]^{-1}
$$

and

$$
\bar{G}_{R}(E)=E D^{-2} \ln \left(E^{2} / D^{2}\right)-i \pi|E| / D^{2},
$$

with $D \simeq 3 t$. Then, using Eq. (43), the $T$ matrix due to an adatom must be of the form

$$
T(E)=\frac{V_{\mathrm{eff}}}{1-V_{\mathrm{eff}} \bar{G}_{R}(E)}=\frac{V_{\mathrm{ad}}^{2}}{E-\epsilon_{\mathrm{ad}}-V_{\mathrm{ad}}^{2} \bar{G}_{R}(E)} .
$$

Since we are considering that $V_{\text {ad }} \gg\left(\epsilon_{\text {ad }},|E|\right)$, we can approximate the $T$ matrix, Eq. (46), by

$$
T(E) \approx-\frac{1}{\bar{G}_{R}(E)},
$$

which is nothing but the $T$ matrix for vacancies. ${ }^{25}$

The transport relaxation time $\tau\left(k_{F}\right)$ (at the Fermi surface) can be calculated using Fermi's golden rule,

$$
\hbar / \tau\left(k_{F}\right)=\pi n_{i}^{c}\left|T\left(\epsilon_{F}\right)\right|^{2} \rho\left(\epsilon_{F}\right),
$$

where $n_{i}^{c}$ is the concentration of impurities per unit cell, and $k_{F}$ and $\epsilon_{F}$ are the Fermi momentum and energy, respectively. From the knowledge of $\tau\left(k_{F}\right)$, the conductivity of graphene follows from Boltzmann's transport equation (see the following section). ${ }^{48}$

\section{B. The Boltzmann approach to dc conductivity using partial-wave expansion}

The above analysis made transparent that the effect of resonant scatterers is equivalent to that of a strong on-site potential (as long as the $T$-matrix formalism is applicable). We can then use the formalism of Sec. II to compute the exact phase shifts in the presence of such a strong potential, from which $\tau\left(k_{F}\right)$ can be obtained. This type of calculations is equivalent, and alternative, to calculations based on the $T$-matrix approach in the lattice, with the appropriate choice of the effective size of the impurity.

A relation between $\tau\left(k_{F}\right)$ and $\sigma(\theta)$ is provided by ${ }^{48}$

$$
1 / \tau\left(k_{F}\right)=n_{i}\left(\mathbf{v}_{k_{F}} \cdot \mathbf{e}_{r}\right) \sigma_{\mathrm{T}},
$$

where $n_{i}$ is the concentration of impurities per unit area, $\mathbf{v}_{k_{F}}$ is the velocity of the electrons at the Fermi surface, $\mathbf{e}_{r}$ is the radial versor in cylindric coordinates, and $\sigma_{T}$ is the total transport cross section: ${ }^{48}$

$$
\begin{aligned}
\sigma_{\mathrm{T}} & =\int_{0}^{2 \pi} d \theta(1-\cos \theta) \sigma(\theta) \\
& =\frac{2}{k} \sum_{m=-\infty}^{\infty} \sin ^{2}\left(\delta_{m}-\delta_{m+1}\right) \equiv \frac{2}{k} \Lambda(k) .
\end{aligned}
$$

The conductivity of a given material follows from Boltzmann's transport equation. The electric current has the general form

$$
\boldsymbol{j}=\frac{g_{s} g_{v} e^{2}}{(2 \pi)^{2}} \int d \mathbf{k} \tau(k) \frac{\partial n_{F}(k)}{\partial \varepsilon_{k}}\left(\mathbf{v}_{k} \cdot \mathbf{E}\right) \mathbf{v}_{k},
$$

where $n_{F}$ is the Fermi distribution function, $\varepsilon_{k}$ is the dispersion of the electron, $\mathbf{v}_{k}$ is the velocity of the particle with momentum $k, \mathbf{E}$ is the external electric field, and $g_{s}$ and $g_{v}$ are the spin and valley degeneracies, respectively. The electron velocity at the Fermi surface reads

$$
\mathbf{v}_{k_{F}}=v_{F} \mathbf{e}_{r}
$$

whereas in the bilayer it has the form

$$
\mathbf{v}_{k_{F}}=\frac{2 v_{F}^{2}}{t_{\perp}} \hbar k_{F} \mathbf{e}_{r},
$$

which depends on the position of the Fermi energy; the quantity $M^{-1}=2 v_{F}^{2} / t_{\perp}$ plays the role of the electron's band mass. The dc conductivity $\sigma_{\mathrm{dc}}$ can be obtained from Ohm's law, $j_{x}=\sigma_{\mathrm{dc}} E_{x}$. Combining Eqs. (51), (52), (53), and (54), the dc conductivity for both monolayer and bilayer graphene has one and the same form, namely,

$$
\sigma_{\mathrm{dc}}=\frac{4 e^{2}}{h} \frac{k_{F}^{2}}{4 n_{i} \Lambda\left(k_{F}\right)},
$$

where the zero-temperature limit has been taken. The importance of Eq. (55) cannot be overemphasized, since it shows that the final dependence of the conductivity on $k_{F}$, and therefore on the electronic density, is controlled by the behavior of $\Lambda\left(k_{F}\right)$, which depends only on the phase shifts $\delta_{m}$; these, in turn, depend on the nature of the scattering potential. Therefore, the exact calculation of the phase shifts emerges as the central theoretical problem regarding the description of the variation of $\sigma_{\mathrm{dc}}$ with the gate voltage for monolayer and bilayer graphene.

\section{Graphene}

For monolayer and bilayer graphene, the electronic doping is controlled by a backgate voltage $V_{g}$. The value of the Fermi momentum depends on the density of electrons and, therefore, also on $V_{g}$. If the dielectric between graphene (or its bilayer) and the backgate is made of silicon oxide and has a width of 
about $300 \mathrm{~nm}$, then we have

$$
k_{F}^{2}=\pi \alpha V_{g},
$$

with $\alpha \simeq 7.2 \times 10^{10} \mathrm{~V}^{-1} \mathrm{~cm}^{-2}$; numerically we have $k_{F}=$ $4.7 \times 10^{-3} \times \sqrt{V_{g}} \AA^{-1}$.

As discussed in Sec. III A, an adsorbed atom or molecule (of specific types) can be described as an effective strong short-range potential. As a consequence, we model the effect of an adsorbed (resonant) chemical species at the surface of graphene by a potential of the form

$$
V(r)=V_{0} \theta(R-r),
$$

where $R$ has to be of the order of $\sim 1 \AA$ and $V_{0} \gg t$. As a limiting behavior, we consider that $V_{0}$ is made arbitrarily large. In the Appendix we discuss the case where the potential is represented by a Dirac $\delta$ function. The latter problem can be solved nonperturbatively, and an effective length scale $R_{\text {eff }}$ emerges in the problem due to an energy cutoff associated with the bandwidth. This effective length scale $\left(R_{\text {eff }}\right)$ is identified with the range $R$ of the potential given above. Both problems lead to the same results for the conductivity of graphene (see later).

In the limit $V_{0} \rightarrow \infty$, the potential defines an impenetrable barrier to the electronic probability flux. For electrons described either by the Schrödinger equation or by the Hamiltonian in Eq. (29), the condition of zero flux for $r<R$ is achieved by imposing that $\Psi(r=R)=0[\Psi(r)$ represents either a scalar or a spinor]. For electrons described by the massless Dirac equation, the latter implies that the wave function has to vanish everywhere and, therefore, cannot be used. In contrast, from Eq. (8) it is clear that the radial flux at $r=R$ can be made 0 if one of the components of the spinor is 0 at $r=R .{ }^{49}$ In conclusion, the correct boundary condition enforcing zero flux at $r=R$ for electrons in monolayer graphene is given by

$$
\Psi_{i}(r=R)=0,
$$

where $\Psi_{i}$, with $i=1,2$, is one of the components of the spinor. Given the presence of two Dirac cones in graphene, it is immaterial which component we choose to obey the condition of Eq. (58), as long as we consider the contributions to the two Dirac cones in the Brillouin zone of the honeycomb lattice.

To satisfy the boundary condition in Eq. (58), we write the wave function describing the electrons being scattered by the barrier as

$$
\Psi_{m}(r, \theta)=A_{1}^{m}\left[\begin{array}{c}
J_{m}(k r) \\
e^{i \theta} J_{m+1}(k r)
\end{array}\right]+A_{2}^{m}\left[\begin{array}{c}
Y_{m}(k r) \\
e^{i \theta} Y_{m+1}(k r)
\end{array}\right] .
$$

Thus, the boundary condition in Eq. (58) implies that

$$
\frac{A_{2}^{m}}{A_{1}^{m}}=-\frac{J_{m}(k R)}{Y_{m}(k R)}
$$

Since for large $r$, the wave function in Eq. (59) must have the general form shown in Eq. (11), it follows that the ratio $A_{2}^{m} / A_{1}^{m}$ has to be interpreted as

$$
\frac{A_{2}^{m}}{A_{1}^{m}}=-\tan \delta_{m},
$$

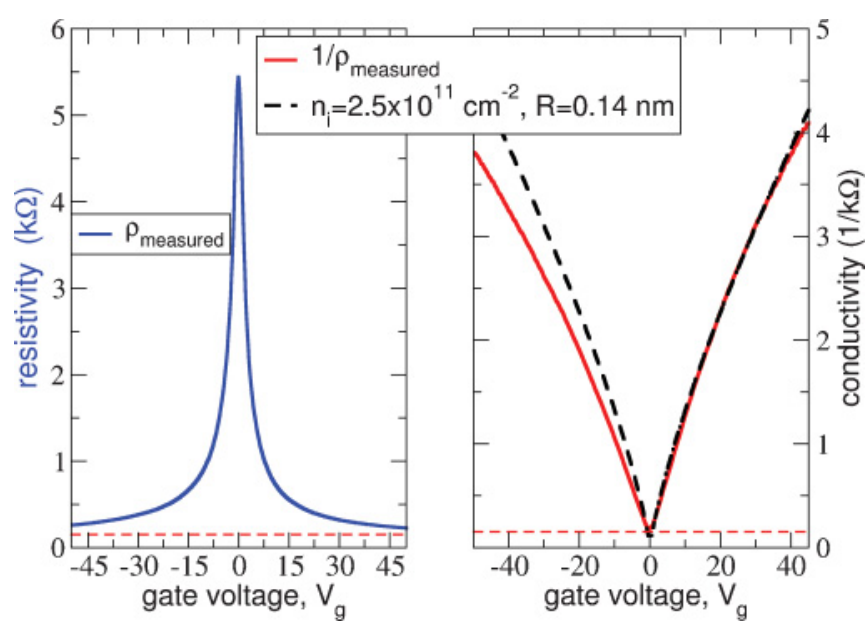

FIG. 5. (Color online) Experimental data on graphene's conductivity. Left: Raw data on a measurement of the resistivity, $\rho_{\text {measured }}$, of an exfoliated graphene sheet. Right: Fit of the conductivity, $\sigma_{\text {sub }}=1 / \rho_{\text {measured }}$, using Eq. (63). The value of $R$ was taken to be of the order of $a_{0}$ and the fit provided an areal density of impurities of $n_{i} \approx 2.5 \times 10^{11} \mathrm{~cm}^{-2}$ (or a concentration $n_{\text {ad }} \approx 5 \times 10^{-5}$ per carbon atom). In both panels, the horizontal dashed (red) line stands for twice the quantum of conductance, that is, $2 e^{2} / h$. (Data from S. V. Morozov et al., ${ }^{50}$ courtesy of A. K. Geim.)

which defines the phase shift $\delta_{m}$. [A comment about the latter result is in order: In graphene, radially symmetric potentials originate phase shifts obeying $\delta_{m}=\delta_{-m-1}$. This can be seen by noting that replacing $m$ by $-m-1$ in Eq. (10) produces another eigenstate of the Dirac Hamiltonian. Equations (60) and (61) show that impenetrable barriers force a different symmetry: $\delta_{m}=\delta_{-m}$.] For backgate voltage values in the range $V_{g} \lesssim 100 \mathrm{~V}$, and considering $R \sim 1 \AA$, we have $R k<1$ (known as the low-energy scattering regime). In this regime, the scattering is dominated by the $s$-wave phase shift; that is, the dominant contribution to $\Lambda(k)$ comes from

$$
\tan \delta_{0}=\frac{J_{0}(k R)}{Y_{0}(k R)} \approx \frac{\pi}{2} \ln ^{-1}(k R),
$$

where Eqs. (20) and (21) have been used. It follows from Eqs. (51) and (62) that the conductivity of graphene obtained from Eq. (55) has the final form ${ }^{25,31,42,51}$

$$
\sigma_{\mathrm{dc}}=\frac{4 e^{2}}{h} \frac{k_{F}^{2}}{2 \pi^{2} n_{i}} \ln ^{2}\left(k_{F} R\right)
$$

Given that the value of $R$ is constrained to be of the order of $1 \AA$, $n_{i}$ is the only fitting parameter. Equation (63) was used to fit the conductivity data ${ }^{50}$ of an exfoliated graphene sheet, as shown in Fig. 5. Because we took the limit $V_{0} \rightarrow \infty$, the computed conductivity does not break electron-hole symmetry. The electron-hole asymmetry shown by the experimental data in Fig. 5 can be attributed to the presence of charge scatterers and/or to the role of the contacts. ${ }^{52}$ If we increase the value of $R$ somewhat, the concentration of impurities needed to fit the data decreases. In Fig. 5 we have chosen to fit the conductivity for a positive gate voltage; it is manifest that Eq. (63) fits the data accurately [dashed (black) curve]. If we had decided to fit the data for negative values of $V_{g}$, the obtained concentration of impurities, $n_{i}$, would have been slightly different. The 
concentration of scatterers is rather small (see caption to Fig. 5) and agrees with the concentration of atomic scale defects estimated via Raman measurements. ${ }^{23}$ This testifies to the strong effect of a few resonant scatterers dilluted in the surface of graphene (similar to atomic vacancies), as discussed in Sec. III A.

The result given by Eq. (63) for the conductivity of monolayer graphene can also be obtained from a model where vacancies act as scattering centers. ${ }^{25}$ In view of the arguments given in Sec. III A, this result comes as no surprise, since the effective local potential created by adsorbed hydrocarbons is much larger than the hopping integral $t$. Numerical simulations of the dc conductivity based on Kubo's formula in the presence of local potentials found a sublinear behavior for a graphene monolayer, ${ }^{53}$ in qualitative agreement with Eq. (63).

Let us now extend the previous analysis to the case of a graphene bilayer.

\section{Graphene bilayer}

Assuming that the dominant source of scattering in graphene is due to strong short-range potentials, then the same must be true for bilayer graphene. As a consequence, a consistent description of electronic scattering in both monolayer and bilayer graphene must use the same scattering potential to explain the measured conductivity in both systems. In the spirit of this work, this means that the scattering potential in Eq. (57) must also be used to compute the conductivity of graphene bilayer.

As in the case of Eq. (59), we seek a wave function in the form of a superposition of Bessel functions of different kinds, which in the present case assumes the form

$$
\begin{aligned}
\Psi_{m}(r, \theta)= & A_{1}^{m}\left[J_{m}(k r)-e^{2 i \theta} J_{m+2}(k r)\right]+A_{2}^{m}\left[Y_{m}(k r)\right. \\
& \left.-e^{2 i \theta} Y_{m+2}(k r)\right]+A_{3}^{m}\left[K_{m}(k r)-e^{2 i \theta} K_{m+2}(k r)\right] .
\end{aligned}
$$

The introduction of the modified Bessel function $K_{m}(k r)$ in Eq. (80) is necessary to satisfy the boundary condition $\Psi(r=R)=0$. We recall that Hamiltonian in Eq. (29) supports evanescent waves at the boundary $r=R$, as discussed in Sec. II B. Furthermore, for large $r, K_{m}(k r)$ decays exponentially, as we can see from Eq. (19). Therefore, at large distances, the behavior of the wave function in Eq. (80) depends only on the form of $J_{m}(k r)$ and $Y_{m}(k r)$, as given by Eqs. (12) and (13). As a consequence, the phase shift $\delta_{m}$ is determined by the ratio $A_{2}^{m} / A_{1}^{m}$; that is, we must have

$$
\frac{A_{2}^{m}}{A_{1}^{m}}=-\tan \delta_{m},
$$

as in the case of electrons in monolayer graphene [see Eq. (61)]. Imposing the boundary condition $\Psi(r=R)=0$ on the wave function (80), we obtain

$$
\begin{aligned}
& 0=A_{1}^{m} J_{m}(k R)+A_{2}^{m} Y_{m}(k R)+A_{3}^{m} K_{m}(k R), \\
& 0=A_{1}^{m} J_{m+2}(k R)+A_{2}^{m} Y_{m+2}(k R)+A_{3}^{m} K_{m+2}(k R),
\end{aligned}
$$

from which follows

$$
\frac{A_{2}^{m}}{A_{1}^{m}}=\frac{J_{m}(k R) K_{m+2}(k R)-J_{m+2}(k R) K_{m}(k R)}{K_{m}(k R) Y_{m+2}(k R)-K_{m+2}(k R) Y_{m}(k R)} .
$$

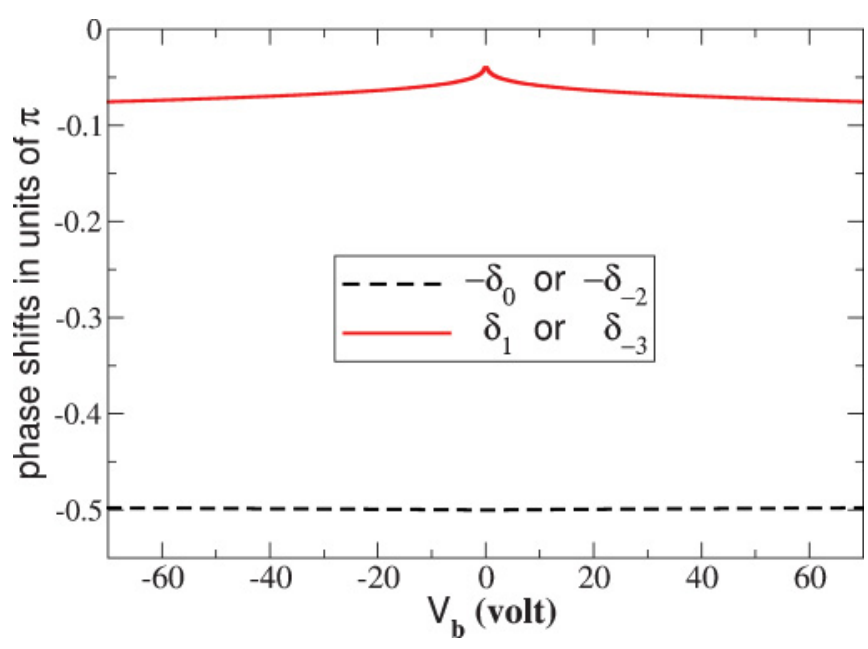

FIG. 6. (Color online) Dependence of the phase shifts $\delta_{0(-2)}$ (solid line) and $\delta_{1(-3)}$ (dashed line) on $V_{g}$, for bilayer graphene with $R=a_{0}$. The differences between the exact expressions in Eq. (65) and the asymptotic values in Eqs. (68) and (69) are not visible to the eye. Other phase shifts are approximately 0 within the same range of $V_{g}$.

Combining Eqs. (65) and (67), the equation for the phase shift $\delta_{m}$ follows at once. Contrary to the case of monolayer graphene, the cross section is no longer dominated by $\delta_{0}$ alone. The asymptotic expansions for $\delta_{0}$ and $\delta_{1}$ are $\left(k_{F} R<1\right)$

$$
\tan \delta_{0}=-\frac{\pi}{2\left(k_{F} R\right)^{2}}\left[\ln \left(k_{F} R / 2\right)+\gamma_{E}-1 / 2\right]^{-1}
$$

and

$$
\tan \delta_{1}=\frac{\pi}{4}\left[\ln \left(k_{F} R / 2\right)+\gamma_{E}-1 / 4\right]^{-1},
$$

where $\gamma_{E}=0.577 \ldots$ is Euler's constant. In addition, we have two more nonzero phase shifts:

$$
\delta_{-2}=\delta_{0} \text { and } \delta_{-3}=\delta_{1} .
$$

These expressions are exact and reflect a symmetry of the eigenstates of Eq. (29) when radially symmetric scalar potentials are considered, namely, $\delta_{m}=\delta_{-m-2}$.

The dependence of $\delta_{0}$ and $\delta_{1}$ on $V_{g}$ is given in Fig. 6. From Eqs. (68)-(70), it follows that $\Lambda\left(k_{F}\right) \simeq 4$. The dc conductivity of bilayer graphene is, therefore, given by

$$
\sigma_{\mathrm{dc}}=\frac{4 e^{2}}{h} \frac{k_{F}^{2}}{16 n_{i}} .
$$

Curiously, the symmetry of the scattering amplitudes combine to make $\Lambda\left(k_{F}\right)$ independent of $k_{F}$ (with an accuracy better than $1 \%$ in the relevant range of $k_{F}$ and $R$ ), making the conductivity proportional to the gate voltage. This result, together with the constant density of states (valid when $|E| \ll t_{\perp}$ ), is at the heart of the exact linear dependence of the conductivity on the gate-voltage. We have used Eq. (71) to fit the conductivity data of an exfoliated bilayer graphene sample, as shown in Fig. 7. The fit provides a concentration of impurities of the order of $n_{i} \approx 4 \times 10^{10} \mathrm{~cm}^{-2}$ (i.e., a concentration of adatoms per carbon atom of about $n_{\mathrm{ad}} \approx 1 \times 10^{-5}$ ). Since in bilayer graphene only two of the four surfaces are exposed to the environment, the $n_{i}$ value found above, being slightly smaller than that found for monolayer graphene, can be interpreted as a manifestation of this fact. 


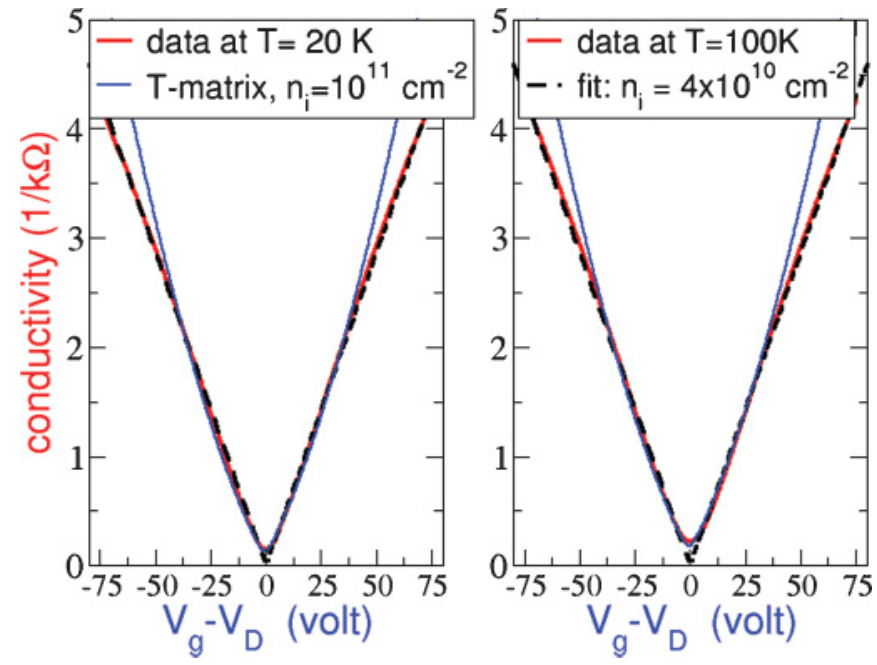

FIG. 7. (Color online) Fit of the conductivity data of bilayer graphene [solid (red) curve] using Eqs. (71) and (74). The fit has only a single parameter, the concentration of impurities. The obtained value is $n_{i} \approx 4 \times 10^{10} \mathrm{~cm}^{-2}$ (concentration $n_{\mathrm{ad}} \approx 1 \times 10^{-5}$ per carbon atom) for Eq. (71) and $n_{i} \approx 1 \times 10^{11} \mathrm{~cm}^{-2}$ (concentration $n_{\text {ad }} \approx 0.25 \times 10^{-5}$ per carbon atom) for the $T$-matrix approach, using a model of pure vacancies, Eq. (74). Left: Data taken at a temperature of $20 \mathrm{~K}$. Right: Conductivity of the same sample at the higher temperature of $100 \mathrm{~K}$. The position of the Dirac point, $V_{D}$, was shifted to 0 in this figure. (Data from S. V. Morison et al.,${ }^{50}$ courtesy of A. K. Geim.)

Within the $T$-matrix approach, the dc conductivity of bilayer graphene has been computed in the past. ${ }^{54,55}$ The impurity concentrations used in those works were far too large to reveal the linear behavior in $V_{g}$ given by Eq. (71). We have already shown that the effect of resonant scatterers can be captured by a model of pure vacancies, using both the $T$-matrix and the partial-wave approaches. We now revisit the $T$-matrix calculation in bilayer graphene ${ }^{54-56}$ and show that, as in the case of the monolayer, a model of pure vacancies in the bilayer also captures the physics of resonant scatterers.

\section{E. $T$-matrix approach for bilayer graphene}

In Refs. 54 and 55, the calculation of the dc conductivity took into account the full band structure of the graphene bilayer. That calculation could distinguish the four carbon atoms in the unit cell. In this section, we assume that vacancies are located at the two carbons that are not coupled by $t_{\perp}$.

In the notation in Refs. 54 and 55, the zero-temperature dc conductivity obtained from Kubo's formula is given by

$$
\begin{aligned}
\sigma_{\mathrm{dc}}= & \frac{8 e^{2}}{\pi h} \int_{0}^{\Lambda^{2}} d\left(k^{2}\right)\left\{\operatorname{Im}\left[g_{A A}^{\mathrm{D}}\left(E_{F}, k\right)\right] \operatorname{Im}\left[g_{B B}^{\mathrm{D}}\left(E_{F}+\delta, k\right)\right]\right. \\
& \left.+\operatorname{Im}\left[g_{A B}^{\mathrm{ND}}\left(E_{F}, k\right)\right] \operatorname{Im}\left[g_{\mathrm{AB}}^{\mathrm{ND}}\left(E_{F}+\delta, k\right)\right]\right\}
\end{aligned}
$$

in the limit $\delta \rightarrow 0$; see Ref. 55 for the definitions of the Green's functions $g(E, k)$. The $k^{2}$ integral can be performed exactly, as explained in Appendix C in Ref. 55. The resulting complicated formula can be approximated by going through the following steps: (i) neglect the real part of the selfenergies, (ii) expand the result in powers of the imaginary part of the self-energies $\Gamma_{a}(\epsilon) \equiv-\operatorname{Im}\left[\Sigma_{a}(\epsilon)\right]$, and (iii) assume that the energies involved fulfill $|\mu|, t_{\perp} \pm|\mu| \gg \Gamma_{A}(\epsilon), \Gamma_{B}(\epsilon)$. The leading term in this expansion yields the approximate formula

$$
\sigma_{\mathrm{dc}} \approx \frac{2 e^{2}}{h} \frac{E_{F}\left(E_{F}+t_{\perp}\right)}{t_{\perp} \Gamma_{B}\left(E_{F}\right)+E_{F}\left[\Gamma_{A}\left(E_{F}\right)+\Gamma_{B}\left(E_{F}\right)\right]} .
$$

This expression is a good approximation for low impurity concentrations and away from the neutrality point, where the condition in step iii breaks down. This result may be further simplified using the relation between the Fermi energy and the density (assuming $n, E_{F}>0$ ) coming from the dispersion relation $E_{F}=\sqrt{\left(t_{\perp} / 2\right)^{2}+\pi\left(\hbar v_{F}\right)^{2} n}-t_{\perp} / 2$, resulting in

$$
\sigma_{\mathrm{dc}}=\frac{2 e^{2}}{h} \frac{\pi\left(\hbar v_{F}\right)^{2} n}{t_{\perp} \Gamma_{B}\left(E_{F}\right)+E_{F}\left[\Gamma_{A}\left(E_{F}\right)+\Gamma_{B}\left(E_{F}\right)\right]},
$$

where $n$ is the electronic density. To the extent that the denominator is independent of $E_{F}$, the conductivity is linear in the density of carriers, $n$, in agreement with the description based on the phase shifts. For low impurity densities, as is the case in exfoliated samples, the difference between the conductivity obtained from the coherent potential approximation and the $T$ matrix is very small except in a tiny region near the neutrality point. Using Eqs. (71) and (74), the data in Fig. 7 can be reasonably fit considering a density of vacancies of $n_{i} \simeq 10^{11} \mathrm{~cm}^{-2}$.

\section{F. Exact amplitudes versus first Born approximation}

The use of the FBA within the semiclassical Boltzmann approach is a common practice in condensed matter. In the present context, the FBA has been employed to investigate the interplay between short-range and long-range scattering. ${ }^{57,58}$ Its use, however, requires the weak scattering condition to be verified. We have seen in Sec. III A that adsorbed atoms in graphene give rise to strong local potentials $V_{0} \gg t$, rendering inappropriate the use of the FBA for a description of scattering due to realistic short-range potentials.

The form of the graphene conductivity [see Eq. (63)] is not peculiar when hard-wall boundary conditions are present; potentials characterized by $\delta$ functions in real space yield equivalent results if exact scattering amplitudes are considered instead of the FBA (see Appendix). Moreover, beyond Boltzmann's kinetic theory, tight-binding calculations for graphene sheets with $\sim 0.02 \mu \mathrm{m}^{2}$ show quantitative agreement with Eq. (63) while, at the same time, displaying qualitative disagreement with the FBA. ${ }^{59}$

To demonstrate that $\delta$ potentials also mimic the effect of strong range potentials, we calculate the exact scattering cross sections using the Lippmann-Schwinger equation, an approach well suited to $\delta$ potentials. (To the best of our knowledge, the case of bilayer graphene has not been considered before.) The calculations are shown in the Appendix and important limiting cases are summarized in Table I.

For monolayer graphene the conductivity due to a $\delta$ potential with strength $V_{0}$ reads

$$
\sigma_{\mathrm{dc}}=\frac{4 e^{2}}{h} \frac{2}{n_{i}}\left[\left(k_{F} / 2 \pi\right) \ln \left(k_{F} R\right)-\frac{\hbar v_{F}}{V_{0}}\right]^{2},
$$

where $R$ is a length scale introduced to regularize the Green's function. The FBA is recovered from Eq. (75) by considering 
TABLE I. The conductivity due to a $\delta$ potential: the (FBA) and the nonperturbative result in the relevant regime $V_{0} \gg$ all scales. For comparison, the hard-disk result is listed. Although for the bilayer both the FBA and the exact calculation give a conductivity proportional to $k_{F}^{2}$, we should note that in the former case the conductivity is proportional to the strength of the potential, and therefore the FBA cannot be trusted in the regime of strong potentials, and the agreement of the two approaches is fortuitous.

\begin{tabular}{lcc}
\hline \hline dc-conductivity & Monolayer & Bilayer \\
\hline$\delta$ & & \\
FBA & Const. & $\sim k_{F}^{2}$ \\
$\quad$ Nonperturbative; large $V_{0}$ & $\sim\left[k_{F} \ln \left(k_{F} R\right)\right]^{2}$ & $\sim k_{F}^{2}$ \\
Hard-disk radius $R$ & $\sim\left[k_{F} \ln \left(k_{F} R\right)\right]^{2}$ & $\sim k_{F}^{2}$ \\
\hline \hline
\end{tabular}

$V_{0}$ smaller than relevant scales, yielding a conductivity that does not depend on the carrier density/gate voltage. In contrast, the strong scattering limit $V_{0} \gg|E|$ gives the same dependence found for the hard-disk model [Eq. (63)] upon the identification of $R$ with the potential range.

The situation is quite different in bilayer graphene, being described by a low-energy theory of massive electrons: both weak and strong scattering regimes yield a conductivity proportional to $k_{F}^{2}$ in the entire carrier density range; the exact result reads

$$
\sigma_{\mathrm{dc}}=\frac{4 e^{2}}{h} \frac{1}{16 n_{i}}\left[1+\left(\frac{8 v_{F}^{2} \hbar^{2}}{V_{0} t_{\perp}}\right)^{2}\right] k_{F}^{2} .
$$

Although for the bilayer both the FBA and the exact calculation result in the conductivity being proportional to $k_{F}^{2}$, we should note that in the former case the conductivity is proportional to the strength of the potential, and therefore the FBA cannot be trusted in the regime of strong potentials, and the agreement on the $k_{F}$ dependence of the two approaches is fortuitous. (We remark that the limitations of the FBA for a description of electronic transport are not exclusive to short-range scatterers and can also be found in Coulomb scatterers..$^{29}$ )

The results of the present and previous sections confirm the intuitive idea that $\delta$ potentials and hard-wall (hard-disk) boundary conditions create the same dependence of $\sigma_{\mathrm{dc}}$ on the Fermi momentum. Remarkably, letting $V_{0} \rightarrow \infty$ in Eqs. (75) and (76) gives precisely Eqs. (63) and (71), respectively, and hence the two models are equivalent with regard to strong short-range potentials.

\section{G. Quantum corrections near the neutrality point}

The Boltzmann approach beyond the FBA provides a good description of the effect of strong short-range scatterers on the transport properties of graphene at finite carrier densities (and for not too high concentrations of resonant impurities). ${ }^{59}$ However, near the neutrality point quantum interference effects become important and a fully quantum calculation is needed to assess dc transport. (For recent reviews on the importance of quantum effects in the transport properties of graphene see Refs. 5 and 60.) In what follows, we present large-lattice, tight-binding numerical calculations in the lowdensity regime and finite (high) impurity concentration limit $n_{\text {ad }} \sim 1 \%$, where quantum corrections due to multiscattering events cannot be ignored.

\section{Monolayer graphene}

We start by extending the monolayer tight-binding Hamiltonian [Eqs. (38) and (39)] to include a finite number $N_{\mathrm{ad}}$ of adsorbed atoms of the same species, binding to carbons placed at (random) positions $\left\{\mathbf{s}_{i}\right\}\left(i=1, \ldots, N_{\mathrm{ad}}\right)$,

$$
\begin{aligned}
\hat{H}_{\mathrm{tb}}= & -t \sum_{n, \delta_{i}}\left|\boldsymbol{R}_{n}, A\right\rangle\left\langle\boldsymbol{R}_{n}+\boldsymbol{\delta}_{i}, B\right|+\text { H.c. } \\
& +\sum_{i=1}^{N_{\mathrm{ad}}}\left[V_{\mathrm{ad}} \mid \mathbf{s}_{i}, \text { ad }\right\rangle\left\langle\mathbf{s}_{i}, C_{i}\right|+\text { H.c. } \\
& \left.\left.+\epsilon_{\mathrm{ad}} \mid \mathbf{s}_{i}, \text { ad }\right\rangle\left\langle\mathbf{s}_{i}, \text { ad }\right|\right],
\end{aligned}
$$

where $C_{i}=A(B)$ for adatoms binding to carbon atoms in the $A(B)$ sublattice. The Kubo formula for the zero-temperature dc-conductivity tensor reads ${ }^{61}$

$$
\sigma_{a b}(E)=\frac{2 \pi \hbar e^{2}}{A} \operatorname{Tr}\left[\hat{v}_{a} \delta\left(E-\hat{H}_{\mathrm{tb}}\right) \hat{v}_{b} \delta\left(E-\hat{H}_{\mathrm{tb}}\right)\right],
$$

where $\hat{v}_{a(b)}$ is the $a(b)$ th component of the velocity operator (defined through the Heisenberg equation of motion for the position coordinate) and $A$ stands for area of graphene.

We evaluate the longitudinal component of the conductivity $\sigma_{x x}$ employing a KPM: details of the calculation are given elsewhere. ${ }^{62}$ The KPM amounts to approximate functions defined in bounded intervals by a truncated sum over polynomials with optimized weights. ${ }^{63}$ To illustrate the change in the transport properties near the neutrality point, we simulate mesoscopic-size square sheets of graphene with $N=10^{6}$ carbon sites. An adequate polynomial expansion of Eq. (78) allows us to perform the simulations with modest computational resources.

We found that the expansion of Eq. (78) in Chebyshev polynomials of the first kind converges for concentrations of resonant impurities, $n_{\text {ad }}=N_{\text {ad }} / N$, above a critical value $n_{\text {ad }}^{*}$ of about $1 \%$ (for $N=10^{6}$ ). We interpret this result as an indication that for $n_{\mathrm{ad}}<n_{\mathrm{ad}}^{*}$, electronic carriers are in the ballistic regime. (Recall that only in diffusive or localized regimes can a thermodynamic conductivity be defined.) The values $n_{\text {ad }} \geqslant n_{\text {ad }}^{*}$ correspond to concentrations of short-range scatterers several orders of magnitude larger than what is found in typical laboratory environments (about $10^{-3} \%$; see previous sections and Ref. 23) but can, in principle, be reached via hydrogenation of graphene on $\mathrm{SiO}_{2} \cdot{ }^{24}$ The critical value $n_{\mathrm{ad}}^{*}$ likely indicates the onset of diffusive behavior, $l \leqslant L$, where $l$ is the mean free path and $L$ denotes the lattice linear size. Thus, in principle it can be lowered by increasing $L$.

Figure 8 shows results for conductivity as function of the carrier density; the latter was obtained by integration of the density of states $\rho(E)$ (shown in Fig. 3), according to

$$
n_{\mathrm{c}}\left(E_{F}\right)=\left(g_{s} / D\right) \int_{0}^{E_{F}} \rho(E) d E,
$$

where $D=N+N_{\text {ad }}$ is the total dimension of the problem. The most peculiar feature in Fig. 8 is the plateau of finite conductivity, due to the formation of a low-energy impurity 


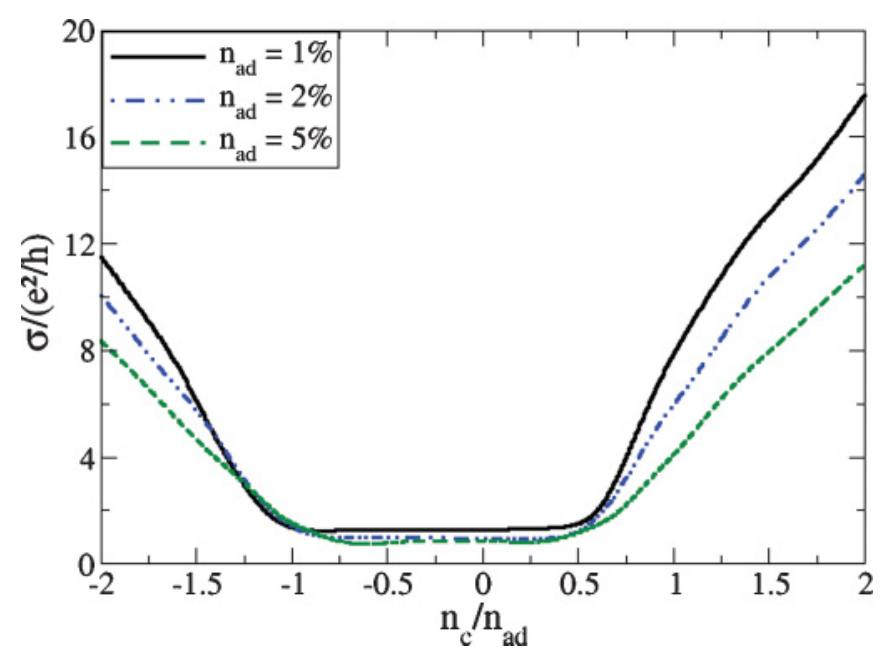

FIG. 8. (Color online) Conductivity as function of the normalized carrier density $n_{\mathrm{c}} / n_{\text {ad }}$ for a monolayer honeycomb lattice with $N=$ $1000 \times 1000$ for different concentrations of adsorbed atoms (periodic boundary conditions and ten realizations of disorder were taken). The tight-binding parameters read $V_{\mathrm{ad}}=2 t$ and $\epsilon_{\mathrm{ad}}=-0.0625 t$.

band (Fig. 3, top), a particular case of disorder-enhanced conductivity. ${ }^{65,66,76}$

The dc conductivity at the neutrality point differs significantly from calculations based on Boltzmann kinetic theory. (1) The conductivity saturates at a low carrier density to a finite value $\sigma_{\min }>0$ around $e^{2} / h$ (the precise value depends on $n_{\mathrm{ad}}$ and sample size), in accordance with theoretical predictions. ${ }^{64}$ The width of the saturation is roughly proportional to the density of adatoms in the probed range of impurity concentration $n_{\text {ad }} \leqslant 5 \%$ (a similar behavior was first reported using a self-consistent approximation to the Green's function of the electrons in the presence of a strong disordered potential ${ }^{25}$ and recently reported in Ref. 20). (2) The conductivity (for a fixed carrier density or energy) is not proportional to $1 / n_{\mathrm{ad}}$. [In fact, a careful inspection of the KPM conductivity data discloses that the latter observation extends to higher carrier densities: (resonant) adsorbate-limited transport in small samples of graphene displays a rich behavior until full diffusive transport is reached.] Both fact 1 and fact 2 above clearly indicate that we are operating outside the applicability of the Boltzmann approach.

Our results, in general, agree well with those reported in Ref. 20 for larger lattices (where $N$ of the order of $10^{8}$ was used). Notwithstanding, we point out some differences concerning the plateau of conductivity minimum: we observe neither peaks within the conductivity plateau (including for $n_{\mathrm{ad}}=5 \%$ ) nor a plateau width of $2 \times n_{\mathrm{ad}}$, as claimed in that work. This could be due to the different methods and system sizes used (although in simulations with a larger lattice, we found no evidence of both effects).

A comment about intervalley scattering in our simulations is in order; Anderson localization induced by intervalley scattering will become experimentally relevant and prevent conductivity saturation only for either very strong disorder (i.e., high defect densities) or exceedingly large samples at very low temperatures. In contrast, our results, and those in Ref. 20 for resonant scatterers, show no evidence of localization even for relatively high amounts of resonant disorder. This suggests that the localization length due to resonant scatterers is far larger than that obtained for an on-site Anderson model, hence allowing for conductivity-induced disorder, $\sigma_{0}>0$, in typical-size graphene samples.

\section{Bilayer graphene}

The tight-binding Hamiltonian for bilayer graphene with resonant impurities reads

$$
\begin{aligned}
\hat{H}_{\mathrm{tb}}^{(\mathrm{BLG})}= & \hat{H}_{\mathrm{tb}}^{(\mathrm{L}=1,2)}+t_{\perp} \sum_{n, \boldsymbol{\delta}_{i}}\left(\left|\boldsymbol{R}_{n}, A_{1}\right\rangle\left\langle\boldsymbol{R}_{n}, B_{2}\right|+\text { H.c. }\right) \\
& +\sum_{\substack{i=1 \\
(L=1,2)}}^{N_{\mathrm{ad}}}\left[V_{\mathrm{ad}} \mid \mathbf{s}_{i}^{L}, \mathrm{ad}\right)\left\langle\mathbf{s}_{i}^{L}, C_{L}\right|+\text { H.c. } \\
& \left.+\epsilon_{\mathrm{ad}}\left|\mathbf{s}_{i}^{L}, \mathrm{ad}\right\rangle\left\langle\mathbf{s}_{i}^{L}, \mathrm{ad}\right|\right],
\end{aligned}
$$

where $\hat{H}_{\mathrm{tb}}^{(\mathrm{L}=1,2)}$ is the Hamiltonian of two uncoupled layers $(L=1,2)$ [see Eq. (77)], the term with $t_{\perp}$ describes electronic interlayer hopping, and the third term accounts for adsorbates binding to carbons in random positions $\left\{\mathbf{s}_{i}^{L}\right\}$ in both layers. We choose $C_{1}\left(C_{2}\right)=A_{2}\left(B_{1}\right)$ to guarantee that adsorbates bind only to carbons with coordination number $z=3$. [The transport properties when adatoms bind to carbons in both sublattices are similar to those of monolayer graphene; see Sec. III A and Fig. 9 (bottom).]

The conductivity of bilayer graphene follows from evaluating the Kubo formula [Eq. (78)] with $\hat{H}_{\mathrm{tb}} \rightarrow \hat{H}_{\mathrm{tb}}^{(\mathrm{BLG})}$. The KPM results (summarized in Fig. 9) resemble those obtained previously for monolayer graphene (Fig. 8), but with important differences. (1) The formation of the impurity band leads to a conductivity minimum about twice the value found for monolayer graphene $\left[\sigma_{\min } \approx e^{2} / h\right.$ (per layer)]. [This fact has been predicted before by coherent potential approximation calculations of disorder in multilayer graphene. ${ }^{54,55}$ See Eqs. (11) and (53) in Refs. 54, and 55, respectively.] (2) For a high impurity concentration, $n_{\mathrm{ad}}=5 \%$, the conducitvity is strongly suppressed before actually forming the plateau; this curious effect is rooted in the opening of a gap in bilayer graphene spectrum, due to the adsorbed species, uncoupling the midgap region from higher energy states (see Fig. 3, bottom, and Fig. 4). In this case, we can then speak of a "conduction gap."

The bottom panel in Fig. 9 compares the conductivity of monolayer and bilayer graphene for $n_{\mathrm{ad}}=5 \%$ : away from the plateau, as carriers have energies similar to or higher than the interlayer coupling $t_{\perp}$, we expect these systems to have comparable conductivities (per graphene layer). Our results indeed confirm the latter point, although we found that for a very high carrier density, $\left|n_{\mathrm{c}}\right| \gtrsim 20 \%$, the conductivity of both systems cannot be compared reliably within our KPM approach: increasing the carrier density up to such values originates carrier energies close to the Von Hove singularities, and strong (spurious) numerical oscillations in the KPM expansion cannot be avoided. In addition, these oscillations behave differently in both systems (in particular, because bilayer graphene has four such singularities), making any comparison difficult. This is the reason why we have presented 

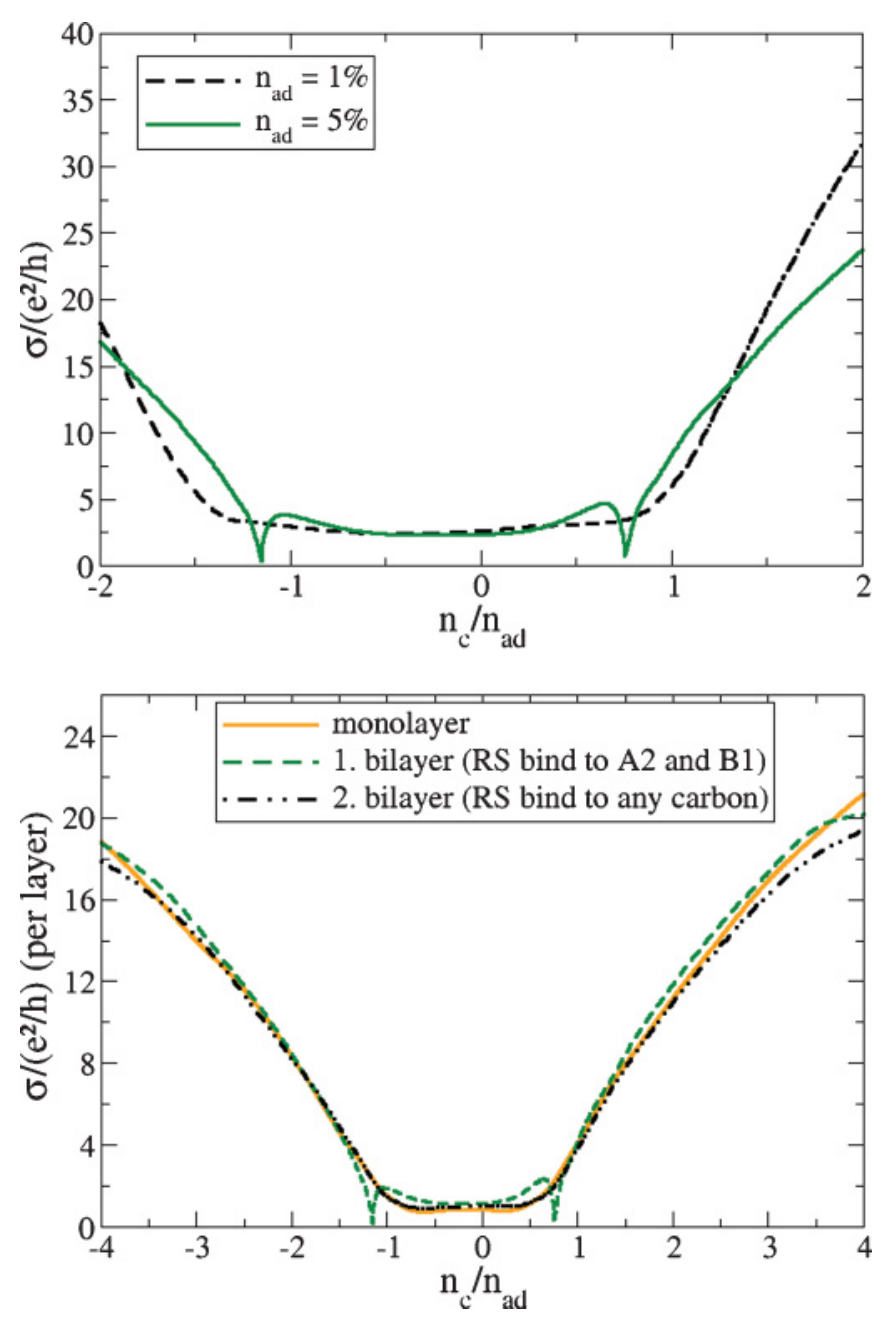

FIG. 9. (Color online) Top: Conductivity as a function of the normalized carrier density $n_{\mathrm{c}} / n_{\text {ad }}$ for a bilayer honeycomb lattice with $N=2 \times 1000 \times 1000$ for different concentrations of adsorbed atoms (periodic boundary conditions and 10 realizations of disorder were taken). The tight-binding parameters read $V_{\text {ad }}=2 t, \epsilon_{\mathrm{ad}}=-0.0625 t$, and $t_{\perp}=0.2 t$. Bottom: Comparison of the conductivity (per layer) of monolayer and bilayer graphene (with $n_{\text {ad }}=5 \%$ in both cases). Two bilayer curves are shown, corresponding to different arrangements of resonant scatterers (RS) as discussed in Sec. (III A): (1) adsorbates binding only to carbons $A_{2}$ and $B_{1}$ and (2) adsorbates forming bonds with carbons in any sublattice. The former situation leads to a supression of the plateau near the edges.

the conductivity for low carrier densities, which also coincides with the most relevant experimental regime.

We finish this section by noting that vacancy-induced disorder leads to effects similar to those reported here, a fact satisfactorily explained by the model of strong short-range scatterers presented in Sec. III A. For vacancies, though, the strong conductivity electron-hole asymmetry (caused by the offset resonant peaks) will not be present.

\section{SCATTERING IN A BIASED BILAYER GRAPHENE}

When $V \neq 0$, electrons in a graphene bilayer are described by Eq. (28). In this case, the energy spectrum develops a Mexican hat form, as represented in Fig. 10, and the spectrum

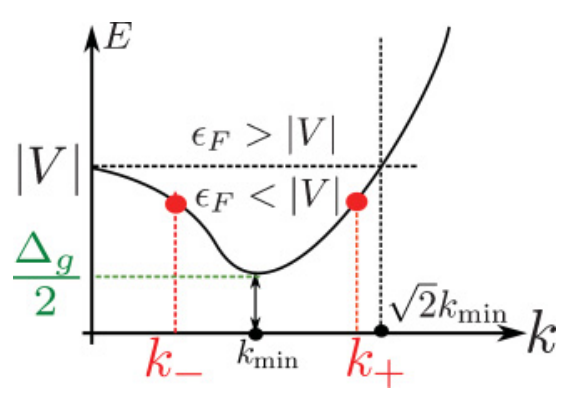

FIG. 10. (Color online) Energy spectrum of a biased graphene bilayer. Several quantities defined in the text are depicted, and $E_{F}$ stands for the Fermi energy. Information on the two regimes $E_{F} \gtrless|V|$ is included. Filled circles represent degenerate states with energy $E=E\left(k_{+}\right)=E\left(k_{-}\right)$, a fact that will have to be taken into account when establishing a scattering theory.

opens up a gap. When the energy of the electrons is lower than $|V|$, the Fermi surface becomes a ring around the Dirac point, with an inner, $k_{-}$, and an outer, $k_{+}$, Fermi radius in momentum space. ${ }^{67,68}$

Therefore, for $E<|V|$, we have two degenerate states with different momentum values. As we show below, the description of scattering in these two regimes, $E \gtrless|V|$, is necessarily different.

The regular eigenstates of Hamiltonian in Eq. (28) in polar coordinates are given by

$$
\Psi_{m}(r, \theta)=\frac{1}{\sqrt{A}}\left[\begin{array}{c}
a_{k} J_{m}(k r) \\
\mp b_{k} J_{m+2}(k r) e^{2 i \theta}
\end{array}\right] e^{i m \theta},
$$

to which corresponds the eigenvalues

$$
E(k)= \pm \sqrt{V^{2}\left(1-\epsilon_{k} / t_{\perp}\right)^{2}+\epsilon_{k}^{2}},
$$

where $\epsilon_{k}=v_{F}^{2} \hbar^{2} k^{2} / t_{\perp}$ is the energy of electrons in bilayer graphene for $V=0$, and the coefficients $a_{k}$ and $b_{k}$ read

$$
\begin{aligned}
a_{k} & =\sqrt{\frac{1}{2}}\left[1+V\left(1-\epsilon_{k} / t_{\perp}\right) / E\right]^{1 / 2}, \\
b_{k} & =\sqrt{\frac{1}{2}}\left[1-V\left(1-\epsilon_{k} / t_{\perp}\right) / E\right]^{1 / 2} .
\end{aligned}
$$

Additionally, the relation $a_{k}^{2} b_{k}^{2}=\epsilon_{k}^{2} /\left(4 E^{2}\right)$ holds.

The density probability flux $J_{\ell}$ is given by Eq. (31), plus an additional term $J_{\ell}^{V}$, reading

$$
J_{\ell}^{V}=2 V \frac{v_{F}^{2} \hbar}{t_{\perp}^{2}} \operatorname{Im} \Psi^{\dagger} \hat{J}_{\ell}^{V} \Psi
$$

where the operator $\hat{J}_{\ell}^{V}$ is given by

$$
\hat{J}_{\ell}^{V}=\left[\begin{array}{cc}
-\partial_{\ell} & 0 \\
0 & \partial_{\ell}
\end{array}\right] \text {. }
$$

Throughout, we consider that electronic carriers have positive energy $E>0$ (the other case follows immediately). Let us establish here some useful relations for later use. The energy gap $\Delta_{g}$ is determined by

$$
\Delta_{g}=2 E\left(k_{\min }\right)=2|V| t_{\perp}\left(V^{2}+t_{\perp}^{2}\right)^{-1 / 2},
$$

where $k_{\min }$ is defined in Eq. (89). Given a state with energy $E$, the two momentum values are obtained from the inversion of 
the energy spectrum, Eq. (82), and are given by the positive roots of the equation

$$
\frac{\epsilon_{k}}{t_{\perp}}=\frac{\Delta_{g}^{2}}{4 t_{\perp}^{2}}[1 \pm f(E)]
$$

with $f(E)=\sqrt{1-\left(1+t_{\perp}^{2} / V^{2}\right)\left(1-E^{2} / V^{2}\right)}$. From Eq. (88) we see that for $E<|V|$ the two roots are real, corresponding to two propagating states, whereas for $E>|V|$, only one root is real, corresponding to a single propagating state; this is consistent with the dispersion depicted in Fig. 10. In the latter regime, the imaginary root is essential to fulfill the scattering boundary conditions, as in the case discussed in Sec. II B. For energy $E=|V|$, we are at the boundary between the two regimes introduced above: $E \gtrless|V|$. In this case, the scattering descriptions below and above $E=|V|$ must provide the same answer. For $E=|V|$ we have $k_{-}=0$ and $k_{+}=\Delta_{g} /\left(\sqrt{2} v_{F} \hbar\right)=\sqrt{2} k_{\min }$; for $E<|V|$ we have a simple relation between $k_{-}$and $k_{+}$, reading

$$
k_{-}=\sqrt{2 k_{\min }^{2}-k_{+}^{2}} \quad \text { and } \quad k_{\min }=\frac{\Delta_{g}}{2 v_{F} \hbar} .
$$

The radial velocity of the electrons at $k_{-}$and $k_{+}$is given by

$$
v_{r}\left(k_{ \pm}\right)=\frac{2 v_{F}^{2} \hbar}{t_{\perp}} \frac{V^{2} f(E)}{t_{\perp} E}\left( \pm k_{ \pm}\right) .
$$

Clearly, the state with momentum $k_{-}$has a negative velocity; the scattering formalism has to take this aspect into account.

Because the regimes $E>|V|$ and $E<|V|$ are distinct, in the sense that the latter case contains two degenerate propagating states, we develop the scattering theory separately for both cases.

\section{A. The $E>|V|$ regime}

For $E>|V|$, the two momenta are $k_{+}=k$ and $k_{-}=$ $i \sqrt{k_{+}^{2}-2 k_{\min }^{2}}=i \kappa$. The latter value originates an evanescent wave at the boundary of the potential. As in the case in Sec. II B, it is simple to show that a wave function of the form

$$
\Psi(\mathbf{r}) \simeq \frac{1}{\sqrt{A}}\left(\begin{array}{l}
a_{k_{x}} \\
b_{k_{x}}
\end{array}\right) e^{i k_{+} x}+\frac{1}{\sqrt{A}}\left(\begin{array}{c}
a_{k} \\
b_{k} e^{2 i \theta}
\end{array}\right) f(\theta) \frac{e^{i k_{+} r}}{\sqrt{r}}
$$

represents an incoming plane wave of momentum $\mathbf{k}_{i}=$ $\left(k_{+}, 0\right) \equiv(k, 0)$ and a scattered cylindrical wave of momentum $\mathbf{k}_{f}=k_{+}(\cos \theta, \sin \theta)$. Note that relative to the case of the unbiased bilayer case, Eq. (97) differs in the presence of the amplitudes $a_{k}$ and $b_{k}$. The scattered radial flux has the usual form $J_{r}=v_{r}(k)|f(\theta)|^{2} / r$, from which the differential cross section follows as $\sigma(\theta)=|f(\theta)|^{2}$. As in Sec. III D, we seek a wave function in the form of a superposition of Bessel functions of different kinds, which in the present case can be written as

$$
\begin{aligned}
\Psi_{m}(r, \theta)= & A_{1}^{m}\left[a_{k} J_{m}(k r)-b_{k} e^{2 i \theta} J_{m+2}(k r)\right] \\
& +A_{2}^{m}\left[a_{k} Y_{m}(k r)-b_{k} e^{2 i \theta} Y_{m+2}(k r)\right] \\
& +A_{3}^{m}\left[a_{\kappa} K_{m}(\kappa r)-b_{i \kappa} e^{2 i \theta} K_{m+2}(\kappa r)\right] .
\end{aligned}
$$

The ratio $A_{m}^{2} / A_{1}^{m}$ reads

$$
\frac{A_{2}^{m}}{A_{1}^{m}}=\frac{a_{k} b_{i \kappa} J_{m}(k R) K_{m+2}(\kappa R)-b_{k} a_{i \kappa} J_{m+2}(k R) K_{m}(\kappa R)}{b_{k} a_{i \kappa} K_{m}(\kappa R) Y_{m+2}(k R)-a_{k} b_{i \kappa} K_{m+2}(\kappa R) Y_{m}(k R)} .
$$

Combining Eqs. (65) and (93), the equation for the phase shift $\delta_{m}$ follows at once. Indeed, the expression for the dc conductivity of electrons with Fermi momentum $k_{+}$is similar to Eq. (55), reading

$$
\sigma_{\mathrm{dc}}=\frac{4 e^{2}}{h} \frac{k_{+}^{2}}{4 n_{i} \Lambda\left(k_{+}\right)} .
$$

In the regime $k_{+} \gg \sqrt{2} k_{\min }$, we have $\kappa \approx k_{+}=k, a_{k} \approx a_{i \kappa}$, and $b_{k} \approx b_{i \kappa}$, and therefore the phase shifts given by Eq. (67) and (93) are essentially identical; that is, we have

$$
\delta_{0} \rightarrow \frac{\pi}{2} \quad\left(k_{+} \gg \sqrt{2} k_{\min }\right) .
$$

As a consequence of Eq. (95), the conductivity is essentially linear in $V_{g}$ at a high electronic density.

When the gate voltage is reduced, bringing the Fermi energy close to $V$, we have $\kappa \rightarrow 0$, but $k_{+} \gtrsim \sqrt{2} k_{\min }$ is finite. In this case, we have

$$
\frac{A_{2}^{m}}{A_{1}^{m}} \rightarrow-\frac{J_{m+2}(k R)}{Y_{m+2}(k R)},
$$

and considering that $k R \lesssim 1$, the $s$-wave phase shift tends to

$$
\delta_{0} \rightarrow-\frac{\pi}{8}\left(k_{\min } R\right)^{4} \quad \text { for } \quad k_{+} \rightarrow \sqrt{2} k_{\min } .
$$

The bias potential acts differently on electron and hole carriers [see Eq. (28)], with the effect that the symmetry relation between phase shifts changes to $\delta_{m}(E, V)=\delta_{-m-2}(-E, V)$. Also, the phase shifts for negative energy carriers (holes) must equal the phase shifts for positive energy carriers (electrons) if the sign of $V$ is reversed.

The dependence of the phase shifts on the gate voltage (that is, on both $k$ and $V$ ) is now more involved. Figure 11 shows the nonzero phase shifts for electrons for the particular case of a weak interlayer potential $V$. Similarly to the unbiased bilayer $(V=0)$ there are four (nonzero) phase shifts, however, as stressed above, the presence of the interlayer potential lifts the degeneracy observed in Fig. 6; in particular, for $|V|>0$ the phase shifts with $m=-1$ and $m=-3$ differ greatly (except for energies very close to $V$ ). On the contrary, the phase shifts $\delta_{0}$ and $\delta_{-2}$ differ significantly only close to the vicinity of $E=V$, where the system approaches the "Mexican hat."

\section{B. The $E<|V|$ regime}

As discussed at the beginning of Sec. IV, in the case $E<$ $|V|$ there are two degenerate propagating states, characterized by $k_{-}$and $k_{+}$. Thus, the matrix element of the potential between these two states is finite, and an incoming particle with a well-defined momentum $\left(k_{-}\right.$or $\left.k_{+}\right)$will be scattered in a superposition of both momenta. This fact requires the modification of the scattering formalism introduced above.

In what follows, we develop the scattering formalism assuming that the incoming electron has momentum $k_{+}$; the case where the incoming electron has momentum $k_{-}$follows immediately, and only the final results are given. 


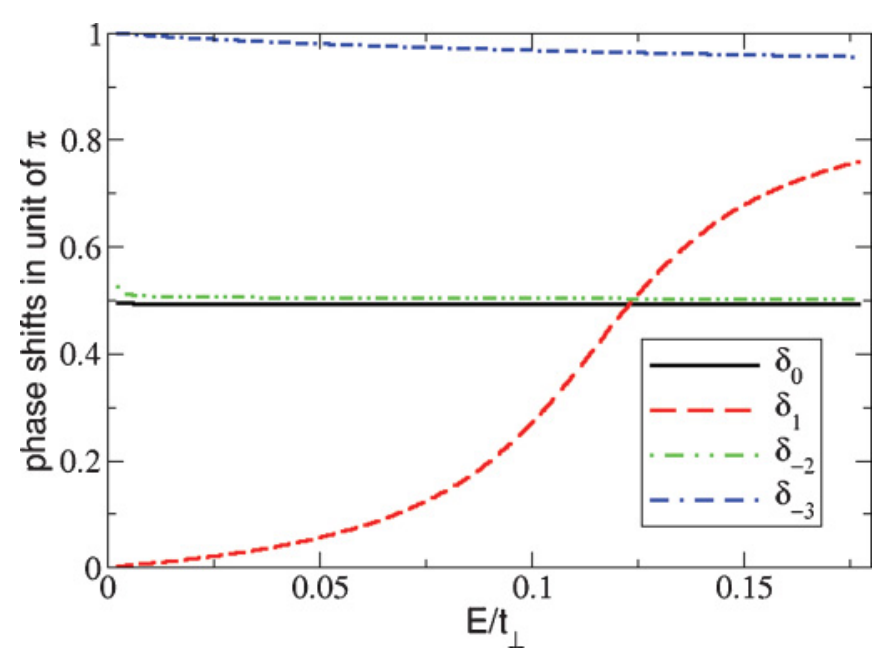

FIG. 11. (Color online) Dependence of nonzero phase shifts $\delta_{m}$ on $E$, for the biased bilayer graphene with $R=a_{0}$ for low electrostatic potential $V=4 \times 10^{-3} t_{\perp}$. The energy range here excludes the interval $\left[4,5\left[\left(10^{-3} t_{\perp}\right)\right.\right.$, for which the energy begins to fall within the "Mexican hat" (Fig. 10). In the vicinity of $E=|V|$, we have $k_{+} \rightarrow \sqrt{2} k_{\text {min }}$ and the $s$-wave phase shift $\delta_{0}$ for electrons (or $\delta_{-2}$ for holes) drops quickly to the value indicated in Eq. (97).

We start by assuming that the total wave function in the presence of the potential, at large distances from it, has the asymptotic form

$$
\begin{aligned}
\Psi(\mathbf{r}) \simeq & \frac{1}{\sqrt{A}}\left[a_{k_{x}} b_{k_{x}}\right] e^{i k_{+} x}+\frac{1}{\sqrt{A}}\left[a_{k_{+}} b_{k_{+}} e^{2 i \theta}\right] f_{++}(\theta) \frac{e^{i k_{+} r}}{\sqrt{r}} \\
& +\frac{1}{\sqrt{A}}\left[a_{k_{-}} b_{k_{-}} e^{2 i \theta}\right] f_{+-}(\theta) \frac{e^{-i k_{-} r}}{\sqrt{r}}
\end{aligned}
$$

where $f_{++}(\theta)$ represents the scattering amplitude considering that the outgoing electron has the same momentum, $k_{+}$, as the incoming one, and $f_{+-}(\theta)$ represents the scattering amplitude considering that the outgoing electron changed its momentum to $k_{-}$. Let us stress again that $E\left(k_{-}\right)=E\left(k_{+}\right)$. Since the velocity of the state with momentum $k_{-}$is negative, the sign of the argument in the exponential of associated cylindrical wave function has to be negative, since these states represent particles propagating backward in time (a positive sign gives a radial incoming flux). The fluxes associated with the first, second, and third terms on the right-hand side of Eq. (98) read

$$
\begin{gathered}
J_{x}^{+}=v_{x}\left(k_{+}\right), \\
J_{r}^{+}=v_{r}\left(k_{+}\right)\left|f_{++}(\theta)\right|^{2} r^{-1},
\end{gathered}
$$

and

$$
J_{r}^{-}=-v_{r}\left(k_{-}\right)\left|f_{+-}(\theta)\right|^{2} r^{-1},
$$

respectively, from which follows the existence of two scattering cross sections, defined as

$$
\sigma_{++}(\theta)=\left|f_{++}(\theta)\right|^{2} \quad \text { and } \quad \sigma_{+-}(\theta)=-\frac{v_{r}\left(k_{-}\right)}{v_{r}\left(k_{+}\right)}\left|f_{+-}(\theta)\right|^{2} \text {. }
$$

Both cross sections must enter in the relaxation time needed to compute the de conductivity.

We now assume that a partial wave in the angular momentum basis of the total wave function has, at large distances from the potential, the form

$$
\begin{aligned}
\Psi_{m}(r, \theta) \simeq & {\left[a_{k_{+}} b_{k_{+}} e^{2 i \theta}\right] \frac{e^{-i\left(k_{+} r-\lambda_{m}-m \theta\right)}}{\sqrt{2 \pi A k_{+} r}} } \\
& +\eta_{m,++} e^{2 i \delta_{m,++}}\left[a_{k_{+}} b_{k_{+}} e^{2 i \theta}\right] \frac{e^{i\left(k_{+} r-\lambda_{m}+m \theta\right)}}{\sqrt{2 \pi A k_{+} r}} \\
& +\eta_{m,+-}\left[a_{k_{-}} b_{k_{-}} e^{2 i \theta}\right] \frac{e^{-i\left(k_{-} r-\lambda_{m}-m \theta\right)}}{\sqrt{2 \pi A k_{-} r}}
\end{aligned}
$$

where $\delta_{m,++}$ is the phase shift of the partial wave $m, 0<$ $\eta_{m,++}<1$ is a real number accounting for the transfer of probability flux to the outgoing momentum channel $k_{-}$, and $0<\left|\eta_{m,+-}\right|^{2}<1$. Conservation of the radial flux for each partial wave $m$ imposes

$$
\eta_{m,++}^{2}+\left|\eta_{m,+-}\right|^{2}=1
$$

Summing over $m$, according to Eq. (11), we obtain $\Psi(\mathbf{r})$ in the form given by Eq. (98), with the scattering amplitudes defined as

$$
\begin{gathered}
f_{++}=\frac{1}{\sqrt{2 \pi i k_{+}}} \sum_{m}\left(\eta_{m,++} e^{2 i \delta_{m,++}}-1\right) e^{i m \theta}, \\
f_{+-}=\frac{1}{\sqrt{2 \pi i k_{-}}} \sum_{m} \eta_{m,+-} e^{i m \theta} .
\end{gathered}
$$

As in Sec. II B, we write the exact partial wave of the full scattering problem, for $r>R$, as

$$
\begin{aligned}
\Psi_{m}(r, \theta)= & A_{1}^{m}\left[a_{k_{+}} H_{m}^{(2)}\left(k_{+} r\right)-b_{k_{+}} H_{m+2}^{(2)}\left(k_{+} r\right) e^{2 i \theta}\right] \\
& +A_{2}^{m}\left[a_{k_{+}} H_{m}^{(1)}\left(k_{+} r\right)-b_{k_{+}} H_{m+2}^{(1)}\left(k_{+} r\right) e^{2 i \theta}\right] \\
& +A_{3}^{m}\left[a_{k_{-}} H_{m}^{(2)}\left(k_{-} r\right)-b_{k_{-}} H_{m+2}^{(2)}\left(k_{-} r\right) e^{2 i \theta}\right] .
\end{aligned}
$$

Expanding Eq. (107) for large $r$ and comparing it with Eq. (103), we see that

$$
\frac{A_{2}^{m}}{A_{1}^{m}}=\eta_{m,++} e^{2 i \delta_{m,++}}, \quad \frac{A_{3}^{m}}{A_{1}^{m}}=\eta_{m,+-} .
$$

Calculation of the differential cross section requires the determination of $\eta_{m,++}, \eta_{m,+-}$, and $\delta_{m,++}$. In the limit $V_{0} \rightarrow$ $\infty$, the boundary condition is $\Psi_{m}(r=R)=0$, leading to

$$
\begin{aligned}
& \frac{A_{2}^{m}}{A_{1}^{m}}=\eta_{m,++} e^{2 i \delta_{m,++}}=\frac{a_{k_{+}} b_{k_{-}} H_{m}^{(2)}\left(k_{+} R\right) H_{m+2}^{(2)}\left(k_{-} R\right)-b_{k_{+}} a_{k_{-}} H_{m+2}^{(2)}\left(k_{+} R\right) H_{m}^{(2)}\left(k_{-} R\right)}{b_{k_{+}} a_{k_{-}} H_{m}^{(2)}\left(k_{-} R\right) H_{m+2}^{(1)}\left(k_{+} R\right)-a_{k_{+}} b_{k_{-}} H_{m+2}^{(2)}\left(k_{-} R\right) H_{m}^{(1)}\left(k_{+} R\right)} \\
& \frac{A_{3}^{m}}{A_{1}^{m}}=\eta_{m,+-}=-a_{k_{+}} b_{k_{+}} \frac{H_{m+2}^{(1)}\left(k_{+} R\right) H_{m}^{(2)}\left(k_{+} R\right)-H_{m}^{(1)}\left(k_{+} R\right) H_{m+2}^{(2)}\left(k_{+} R\right)}{b_{k_{+}} a_{k_{-}} H_{m}^{(2)}\left(k_{-} R\right) H_{m+2}^{(1)}\left(k_{+} R\right)-a_{k_{+}} b_{k_{-}} H_{m+2}^{(2)}\left(k_{-} R\right) H_{m}^{(1)}\left(k_{+} R\right)}
\end{aligned}
$$




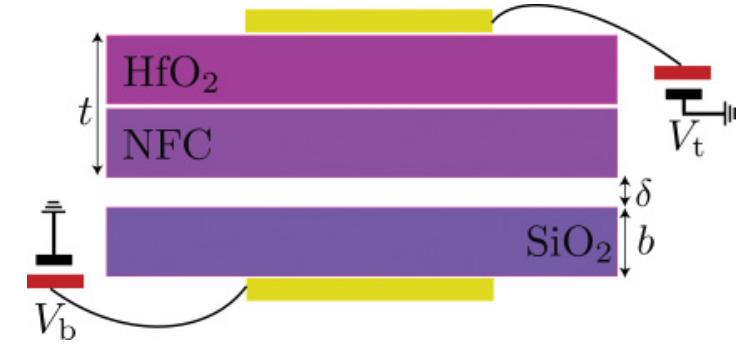

FIG. 12. (Color online) Capacitor geometry for dual-gate transistor. ${ }^{69}$ The figure is self-explanatory. Values of the several quantities are $\delta=3.4 \AA, b=300 \mathrm{~nm}$, and $t=20 \mathrm{~nm}$. $V_{\mathrm{t}}$ and $V_{\mathrm{b}}$ stand for the top and bottom gate potentials, respectively.

Although not immediately obvious, the parameters $\eta_{m,++}$ and $\eta_{m,+-}$, as given by Eqs. (109) and (110), obey the flux conservation relation in Eq. (104). When the Fermi energy $E_{F}$ approaches the energy $E=|V|$ from below, we have $k_{-} \rightarrow 0$. In this limit, we find

$$
\begin{aligned}
\eta_{0,++} e^{2 i \delta_{0,++}} \rightarrow- & -\frac{H_{2}^{(2)}\left(k_{+} R\right)}{H_{2}^{(1)}\left(k_{+} R\right)}, \\
\eta_{m,+-} & \rightarrow 0,
\end{aligned}
$$

as it should. Since $k_{+} R \lesssim 1$, it follows from Eq. (111) that

$$
\delta_{0,++} \rightarrow-\frac{\pi}{32}\left(k_{+} R\right)^{4},
$$

which, for $\delta_{0,++}$, gives the same result found in Eq. (97).

The above results hold for an incoming electron with momentum $k_{+}$; when the electron has momentum $k_{-}$we have to consider the cross sections

$$
\sigma_{--}(\theta)=\left|f_{--}(\theta)\right|^{2} \quad \text { and } \quad \sigma_{-+}(\theta)=-\frac{v_{r}\left(k_{+}\right)}{v_{r}\left(k_{-}\right)}\left|f_{-+}(\theta)\right|^{2},
$$

whose amplitudes are given by the right-hand side of Eqs. (105) and (106), respectively, upon interchanging $k_{+}$with $k_{-}$.

\section{C. de conductivity of a biased bilayer graphene}

As discussed in Sec. III D, calculation of the de conductivity requires the computation of the exact phase shifts. We start by studying the behavior of the $s$-wave phase shift as a function of the Fermi momentum for a biased graphene bilayer.

In the biased bilayer, the ability of independently tuning the electronic density and the value of the gap $\Delta_{g}$ requires the use of two gates, a bottom and a top gate, as shown in Fig. 12. The electric field in the top-gate dielectric is $(e>0)$

$$
E_{\mathrm{t}}=\frac{e n_{\mathrm{t}}}{\epsilon_{\mathrm{t}} \epsilon_{0}}
$$

and that in the bottom-gate dielectric is

$$
E_{\mathrm{b}}=\frac{e n_{\mathrm{b}}}{\epsilon_{\mathrm{b}} \epsilon_{0}},
$$

where $n_{\mathrm{t}}$ and $n_{\mathrm{b}}$ are the electronic density in the top and bottom gate, respectively, and $\epsilon_{\mathrm{t}}$ and $\epsilon_{\mathbf{b}}$ are the relative permittivity of the top- and bottom-gate dielectric, respectively. Charge neutrality requires that the total amount of charge accumulated in the bilayer is $-e n=-e\left(n_{\mathrm{t}}+n_{\mathrm{b}}\right)$. The electrostatic potential difference between the top gate and the bilayer is $V_{\mathrm{t}}=t E_{\mathrm{t}}$, whereas between the bottom gate and the bilayer it is $V_{\mathrm{b}}=b E_{\mathrm{b}}$. It follows from Eqs. (115) and (116) that

$$
V_{\mathrm{b}}=b \frac{e n_{b}}{\epsilon_{\mathbf{b}} \epsilon_{0}}=\frac{b e n}{\epsilon_{\mathbf{b}} \epsilon_{0}}-\frac{b \epsilon_{\mathrm{t}}}{t \epsilon_{\mathbf{b}}} V_{\mathrm{t}} .
$$

Inverting Eq. (117), the total electronic density in the bilayer is given by

$$
n=V_{\mathrm{b}} \frac{\epsilon_{\mathrm{b}} \epsilon_{0}}{b e}+\frac{\epsilon_{0} \epsilon_{\mathrm{t}}}{e t} V_{\mathrm{t}} .
$$

When $n$ is positive, the bilayer is doped with electrons; when $n$ is negative the system is doped with holes. Finally, the electrostatic potential difference between the two graphene layers in the bilayer is given by

$$
\Delta V=\left(E_{\mathrm{b}}-E_{\mathrm{t}}\right) \delta=\frac{n e \delta}{\epsilon_{\mathbf{b}} \epsilon_{0}}-\left(\frac{\epsilon_{\mathrm{t}}}{\epsilon_{\mathbf{b}}}+1\right) \frac{\delta}{t} V_{\mathrm{t}},
$$

where $\delta=3.4 \AA$ is the interlayer distance (we are ignoring screening effects, ${ }^{36,37,39}$ which are not important for small $V_{\mathrm{t}}$ ). The variable $V$ introduced in Eq. (26) relates to $\Delta V$ as $2 V=$ $\Delta V$. Taking typical values for dual-gate bilayer transistors, ${ }^{69}$ we have $\epsilon_{\mathrm{SiO}_{2}}=3.9, \epsilon_{\mathrm{HfO}_{2}}=25, \epsilon_{\mathrm{NFC}}=2.4, b=300 \mathrm{~nm}$, and $t=20 \mathrm{~nm}$ (both dielectrics, $\mathrm{HfO}_{2}$ and $\mathrm{NFC}$, have about the same width). The relative permittivity of $\epsilon_{t}$ is

$$
\epsilon_{\mathrm{t}}=\frac{2 \epsilon_{\mathrm{HfO}_{2}} \epsilon_{\mathrm{NFC}}}{\epsilon_{\mathrm{HfO}_{2}}+\epsilon_{\mathrm{NFC}}} .
$$

In working devices, ${ }^{69}$ we have $\left|V_{\mathrm{b}}\right| \lesssim 70 \mathrm{~V}$ and $\left|V_{\mathrm{t}}\right| \lesssim 4 \mathrm{~V}$.

The calculation of dc conductivity follows, as before, from Boltzmann's transport theory. In the regime $E>|V|, \sigma_{\mathrm{dc}}$ is still given by Eq. (55), but with the phase shifts determined from Eq. (93). When $E<|V|$, there are two scattering channels and this implies that the resulting formula for $\sigma_{\mathrm{dc}}$ differs somewhat from that given in Eq. (55), reading

$$
\sigma_{\mathrm{dc}}=\frac{4 e^{2}}{h} \frac{1}{2}\left[\frac{k_{+}}{n_{i} \sigma\left(k_{+}\right)}+\frac{k_{-}}{n_{i} \sigma\left(k_{-}\right)}\right],
$$

where $\sigma\left(k_{ \pm}\right)$is defined as

$$
\sigma\left(k_{ \pm}\right)=\int_{0}^{2 \pi}\left[\sigma_{ \pm,+}(\theta)+\sigma_{ \pm,-}(\theta)\right](1-\cos \theta) .
$$

Inserting the expressions for the differential cross sections [Eqs. (102) and (114)] and performing the integral yields

$$
\begin{aligned}
\sigma\left(k_{ \pm}\right)= & \frac{1}{k_{ \pm}} \operatorname{Re} \sum_{m}\left[\left|\eta_{m, \pm \pm} e^{2 i \delta_{m, \pm \pm}}-1\right|^{2}\right. \\
& -\left(\eta_{m, \pm \pm} e^{2 i \delta_{m, \pm \pm}}-1\right)\left(\eta_{m+1, \pm \pm} e^{-2 i \delta_{m+1, \pm \pm}}-1\right) \\
& \left.-\frac{k_{ \pm}}{k_{\mp}} \frac{v_{r}\left(k_{\mp}\right)}{v_{r}\left(k_{ \pm}\right)}\left(\left|\eta_{m, \pm \mp}\right|^{2}-\eta_{m, \pm \mp} \eta_{m+1, \pm \mp}^{*}\right)\right] .
\end{aligned}
$$

The formulas for $\sigma_{--}$and $\sigma_{-+}$are identical and thus are not presented. The dc conductivity follows from the determination of the Fermi momentum, given the carrier density in the bilayer, which in turn depends on both gates as given by Eq. (118). The relation $k_{F}^{2}=\pi n$ (valid for various two-dimensional systems) must be adapted to take into account the degeneracy of the spectrum (Fig. 10) and reads

$$
k_{F}^{+}=\sqrt{\frac{\pi}{2} n+k_{\min }^{2}},
$$




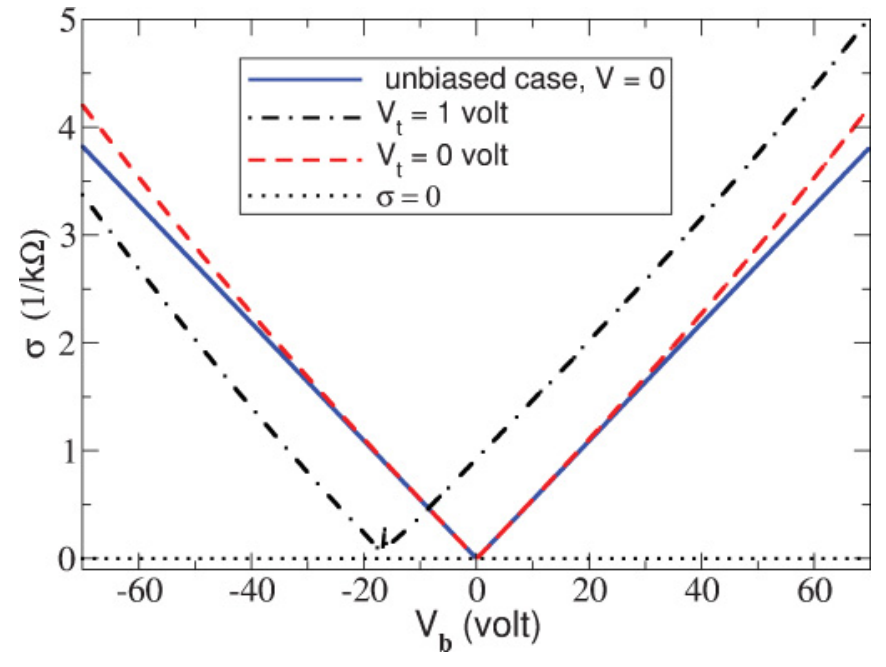

FIG. 13. (Color online) Dependence of the biased bilayer dc conductivity on the back-gate potential $V_{\mathrm{b}}$ (values of $V_{\mathrm{t}}$ are indicated). The solid line shows the dc conductivity for the unbiased case $V=0$, for comparison.

and the other propagating state $k_{F}^{-}$relates to $k_{F}^{+}$according to Eq. (89).

Figure 13 shows the dc conductivity as function of the back gate for fixed values of $V_{\mathrm{t}}$. As the back gate $V_{\mathrm{b}}$ is varied, the gap $\Delta_{g}$ and the Fermi energy change; for a small window, width $\sim 1 \mathrm{~V}$, around $V_{\mathrm{b}} \simeq-17 \mathrm{~V}$ the system moves into the regime $E<|V|$ and the expression in Eq. (124) must be used to determine the carrier energy. In this energy regime, $k_{F}$ is bounded according to $\sqrt{2} k_{\min } \geqslant k_{F} \geqslant k_{\min }$, and hence the value $k_{F}=0$ is forbidden; as a consequence, and at odds with the unbiased bilayer, the minimum conductivity is not exactly 0 , having a value of $\sigma_{\min } \simeq 3 e^{2} / h$ for $V_{\mathrm{t}}=1 \mathrm{~V}$.

\section{CONCLUSION}

In the early studies of transport in graphene, charged impurities located in the substrate seemed to explain the measured conductivity. Recent experiments, however, suggest another possibility. ${ }^{23,24}$ While there is a consensus that electron and hole puddles, induced by charged impurities, dominate the landscape near the neutrality point, away from this point, adsorbed hydrocarbons, at the surface of graphene, may be the limiting factor in de transport.

In the present paper, we have established an intuitive theoretical picture of scattering due to resonant scattering originated by adatoms. Although resonant scatterers have been studied before (first in Refs. 41 and 42 and, more recently, in Ref. 20), we have established the first coherent picture of resonant-scattering limited dc transport valid for both monolayer and bilayer graphene.

Section III A reviews the electronic structure of monolayer graphene and presents, for the first time, the density of states of bilayer graphene with resonant contaminants. Despite the distinct electronic structure of pristine monolayer and bilayer graphene, this section shows that resonant adatoms lead to the same effect in both systems: the emergence of resonant peaks in the vicinity of the Dirac point, a situation reminiscent of vacancy induced disorder. ${ }^{26,44}$ Using a simple tight-binding toy model, resonant adatoms are seen to be reliably mimicked by a particular class of short-range scatterers, that is, those having an intrinsic energy much higher than typical graphene energies. This fact motivates the subsequent study of $\mathrm{dc}$ transport using strong short-range potentials in a continuum formulation (Secs. III C and III D).

Section III shows that the typical dependence of conductivity with the electronic density in the monolayer (sublinear dependence) and bilayer (linear dependence) systems can be explained assuming resonant scatterers alone. The comparison with experimental data bears out the agreement with dc-transport experiments performed in exfoliated fewlayer graphene films, hence providing further strength to the resonant-scatterer hypothesis. To justify the robustness of a continuum-model approach based on strong short-range scatterers as prototypes of real resonant adsorbates, we have calculated the semiclassical conductivity due to two types of strong local potentials (hard-disk and $\delta$ potentials), finding perfect agreement between the two methods (partial-wave analysis and Lippmann-Schwinger equation, respectively) and tested the validity of the long-wavelength limit (on the basis of the continuum formulation) against numerical lattice calculation using a $T$-matrix approach (Sec. III E).

Section III F demonstrates the incorrectness of the widely used FBA within the semiclassical (Boltzmann) approach, in the context of short-range disorder, and the need to compute the electronic scattering amplitudes as accurately as possible, hence, clarifying an issue overlooked in the graphene literature. Section III G presents the Kubo dc conductivity evaluated numerically with a KPM; from this calcution, the breakdown of the semiclassical picture close to neutrality, in the regime of a high concentraton of impurities, is clearly observed. Here, the case of bilayer graphene is addressed for the first time, with the results showing that a "conduction gap" takes place for selective adsorbate bonding, due to a strong supression of the conducitivy in the surroundings of the resonant impurity band.

Finally, due to its importance for technological applications, scattering in the bilayer graphene with a gap in the sectrum is studied in Sec. IV, by extending the well-established partial-wave method (Sec. II) to describe scattering in the biased bilayer graphene. Such a scattering theory has never been developed before (to the best of our knowledge) and can be easily adapted to tackle other physical scenarios requiring the need for computing scattering amplitudes when the energy dispersion relation is degenerate.

We are confident that our results help to elucidate the electronic transport properties of this remarkable two-dimensional material.

Note added: After submission of this work for publication, we become aware of a paper ${ }^{77}$ which also discusses the effect of resonant scatterers on the dc conductivity of single-layer and bilayer graphene, with results that are consistent with ours.

\section{ACKNOWLEDGMENTS}

A.F. acknowledges FCT Grant No. SFRH/BPD/65600/ 2009. E.R.M. acknowledges partial financial support by NSF Grant No. DMR 1006230. A.H.C.N. acknowledges Grant 
No. DE-FG02-08ER46512. Discussions with A. K. Geim are acknowledged.

\section{APPENDIX}

In this appendix, starting from the low-energy continuum theory, we derive the nonperturbative semiclassical dc conductivity of monolayer and bilayer graphene with short-range scatterers. This calculation requires the solution of the twodimensional scattering problem, where a massless fermion with incident momentum $\mathbf{p}=\hbar \mathbf{k}$ is brought to interaction with an impurity. We model the potential of the impurity by a $\delta$ function, $V_{d}=V_{0} \delta(\mathbf{r})$. Following standard methods, the formal solution of $\left(H_{0}+V_{d}-E\right) \Psi_{\mathbf{k}}=0$ can be written as,

$$
\Psi_{\mathbf{k}}=\phi_{\mathbf{k}}+\hat{G}_{0} \hat{V}_{d} \Psi_{\mathbf{k}}
$$

where $H_{0}$ is the low-energy Hamiltonian of graphene; $\phi_{\mathbf{k}}$ is the solution of the free problem $\left(H_{0}-E\right) \phi_{\mathbf{k}}=0$ and describes the state of the incident particles. Here, $H_{0}$ refers to the Hamiltonian obtained from expansion of the graphene dispersion around the $\mathbf{K}$ point (the calculation involving the remaining valley is equivalent). The resolvent is given by $\hat{G}_{0}=1 /\left(E+i 0^{+}-H_{0}\right)$, and the energy includes a small positive imaginary part $i 0^{+}$

$$
\phi_{\mathbf{k}}(\mathbf{r})=u_{\mathbf{k}}^{(\lambda)} e^{i \mathbf{k} \cdot \mathbf{r}}
$$

with

$$
u_{\mathbf{k}}^{(\lambda)}=\frac{1}{\sqrt{2 A}}\left(\begin{array}{c}
1 \\
s e^{i \lambda \theta_{\mathbf{k}}}
\end{array}\right) .
$$

The Berry phase is $\varphi_{B} \equiv \pi \lambda$ and equals $\pi$ for monolayer graphene, whereas for bilayer graphene its value is $2 \pi$ [compare with Eq. (3)]. The second component of $u_{\mathbf{k}}$ includes the sign $s= \pm$ of the electronic carrier charge and $\theta_{\mathbf{k}} \equiv \arctan \left(k_{y} / k_{x}\right)$. Switching Eq. (A1) to the position representation, we obtain the Lippmann-Schwinger equation,

$$
\Psi_{\mathbf{k}}(\mathbf{r})=\phi_{\mathbf{k}}(\mathbf{r})+\int d^{2} \mathbf{r}^{\prime} G_{0}\left(\mathbf{r}-\mathbf{r}^{\prime}\right) V\left(\mathbf{r}^{\prime}\right) \Psi_{\mathbf{k}}\left(\mathbf{r}^{\prime}\right) .
$$

In the latter equation, $G_{0}\left(\mathbf{r}-\mathbf{r}^{\prime}\right)=\left\langle\mathbf{r}\left|\left(E+i 0^{+}-H_{0}\right)^{-1}\right| \mathbf{r}^{\prime}\right\rangle$ is the Green function of the problem.

Monolayer graphene has $H_{0}=\hbar v_{F} \boldsymbol{\sigma} \cdot \hat{\mathbf{p}}$ and the Fourier transform of the Green function obeys

$$
\left(E+i 0^{+}-\boldsymbol{\sigma} \cdot \mathbf{p}\right) G_{0}(\mathbf{p})=1,
$$

where $G_{0}(\mathbf{p})=\int d \mathbf{r} \exp (-i \mathbf{p} \cdot \mathbf{r}) G_{0}(\mathbf{r})$ (note that to simplify notation, we have set $\hbar=1$ and $\left.v_{F}=1\right)$. Inverting the $2 \times 2$ matrix on the left-hand side of Eq. (A5), we arrive at

$$
\begin{gathered}
G_{0}(\mathbf{p})=g_{1}(\mathbf{p})(E+\boldsymbol{\sigma} \cdot \mathbf{p}), \\
g_{1}(\mathbf{p})=1 /\left[E^{2}-p^{2}+i 0^{+}\right] .
\end{gathered}
$$

The calculations for $E>0$ and $E<0$ are similar, and to be specific we focus on the former situation. Indeed, the inclusion of a small imaginary part from positive values $i 0^{+}$amounts to consider outgoing waves (see below). We write $E=k$ and evaluate the Green function in the real-space representation,

$$
\begin{aligned}
G_{0}\left(\mathbf{r}-\mathbf{r}^{\prime}\right) & =\frac{1}{4 \pi^{2}}(E-i \boldsymbol{\sigma} \cdot \nabla) \int d^{2} \mathbf{p} e^{i \mathbf{p} \cdot\left(\mathbf{r}-\mathbf{r}^{\prime}\right)} g_{1}(\mathbf{p}) \\
& =-\frac{i}{4}(k-i \boldsymbol{\sigma} \cdot \nabla) H_{0}^{(1)}\left(k\left|\mathbf{r}-\mathbf{r}^{\prime}\right|\right),
\end{aligned}
$$

where $H_{n}^{(1)}\left(k\left|\mathbf{r}-\mathbf{r}^{\prime}\right|\right)$ is the Hankel function of the first kind of order $n$, whose asymptotic form is that of outgoing cylindrical waves [see Eq. (A24)]. The Hankel function obeys $\partial_{x} H_{0}^{(1)}(x)+$ $H_{1}^{(1)}(x)=0$; hence,

$$
\boldsymbol{\sigma} \cdot \nabla H_{0}^{(1)}\left(k\left|\mathbf{r}-\mathbf{r}^{\prime}\right|\right)=-k H_{1}^{(1)}\left(k\left|\mathbf{r}-\mathbf{r}^{\prime}\right|\right) \sigma_{\theta},
$$

where we have introduced the matrix,

$$
\sigma_{\theta} \equiv\left(\begin{array}{cc}
0 & e^{-i \theta} \\
e^{i \theta} & 0
\end{array}\right),
$$

and the angle $\theta \equiv \theta\left(\mathbf{r}, \mathbf{r}^{\prime}\right)$ is defined by $\left(\mathbf{r}-\mathbf{r}^{\prime}\right) /\left|\mathbf{r}-\mathbf{r}^{\prime}\right|=$ $(\cos \theta, \sin \theta)^{T}$. Combining Eqs. (A10) and (A9), we have, at once,

$$
G_{0}\left(\mathbf{r}-\mathbf{r}^{\prime}\right)=-\frac{i k}{4}\left[H_{0}^{(1)}\left(k\left|\mathbf{r}-\mathbf{r}^{\prime}\right|\right)+i \sigma_{\theta} H_{1}^{(1)}\left(k\left|\mathbf{r}-\mathbf{r}^{\prime}\right|\right)\right] .
$$

The derivation of the Green function of bilayer graphene follows identical steps. We write the free Hamiltonian as $H_{0}=$ $-\left(v_{F}^{2} \hbar^{2} / t_{\perp}\right) \boldsymbol{\sigma} \cdot \mathbf{D}$, with $\mathbf{D}=\left(\partial_{x}^{2}-\partial_{y}^{2}, 2 \partial_{x} \partial_{y}\right)^{T}$. As before, we set $\hbar$ and $v_{F}$ temporarily equal to the unit; the Fourier transform of bilayer Green function reads

$$
G_{0}(\mathbf{p})=g_{2}(\mathbf{p})[E+\gamma \boldsymbol{\sigma} \cdot \tilde{\mathbf{D}}(\mathbf{p})],
$$

where $\gamma \equiv 1 / t_{\perp}, E=\gamma k^{2}, \tilde{\mathbf{D}}(\mathbf{p})=\left(p_{x}^{2}-p_{y}^{2}, 2 p_{x} p_{y}\right)^{T}$, and

$$
g_{2}(\mathbf{p})=\frac{1}{2 E}\left[\frac{1}{E-\gamma p^{2}+i 0^{+}}+\frac{1}{E+\gamma p^{2}+i 0^{+}}\right] \text {. }
$$

Since $H_{0}$ is quadratic in momentum operators, $g_{2}$ resembles a nonrelativistic propagator. Again, we focus on the case of electrons $(\gamma>0)$,

$$
G_{0}\left(\mathbf{r}-\mathbf{r}^{\prime}\right)=\frac{\gamma}{4 \pi^{2}}\left(k^{2}-\sigma \cdot \mathbf{D}\right) \int d^{2} \mathbf{p} e^{i \mathbf{p} \cdot\left(\mathbf{r}-\mathbf{r}^{\prime}\right)} g_{2}(\mathbf{p}) .
$$

The contribution to the integrand of Eq. (A15) with poles in the real axis can be simplified using

$$
\frac{1}{k^{2}-p^{2}+i 0^{+}}=\frac{i \pi}{2 k}[\delta(p+k)+\delta(p-k)]+\mathrm{P} . \mathrm{V} \cdot \frac{1}{k^{2}-p^{2}} \text {. }
$$

Performing the integral in Eq. (A15) yields

$$
\begin{aligned}
G_{0}\left(\mathbf{r}-\mathbf{r}^{\prime}\right)= & \frac{1}{8 \gamma k^{2}}\left(k^{2}-\sigma \cdot \mathbf{D}\right)\left[-i H_{0}^{(1)}\left(k\left|\mathbf{r}-\mathbf{r}^{\prime}\right|\right)\right. \\
& \left.+\frac{2}{\pi} K_{0}\left(k\left|\mathbf{r}-\mathbf{r}^{\prime}\right|\right)\right] .
\end{aligned}
$$

The first term in brackets describes scattered waves in two dimensions, whereas the modified Bessel function $K_{0}$ describes evanescent waves (recall that $k \rightarrow i k$ is a solution of $H_{0}$ with the same energy). For short-range potentials the main contribution to the scattering amplitude comes from evaluating Eq. (A4) within the region where $\left|\mathbf{r}-\mathbf{r}^{\prime}\right| \gg 1$ and hence $K_{0}$ will not contribute (see later). 
In what follows, we compute the nonperturbative scattering amplitude for monolayer and bilayer graphene, which will be needed for the calculation of the dc conductivity in these systems.

\section{Nonperturbative amplitude for monolayer graphene}

Inserting the expression of the potential $V_{d}=V_{0} \delta(\mathbf{r})$ in the Lippmann-Schwinger equation [Eq. (A4)] and performing the spatial integration results in

$$
\Psi_{\mathbf{k}}(\mathbf{r})=\phi_{\mathbf{k}}(\mathbf{r})+V_{0} G_{0}(\mathbf{r}) \Psi_{\mathbf{k}}(0),
$$

which is ill defined because putting $\mathbf{r}=0$ yields a divergence, namely, $\Psi_{\mathbf{k}}(0)=\phi_{\mathbf{k}}(0)+(\infty)$. This stems from the singularity of $G_{0}(\mathbf{r})$ [Eq. (A12)] at the origin $\mathbf{r}=0$, a common situation in field theories. The only way of curing this divergence is by means of renormalization. ${ }^{70}$ Let us explicitly describe this procedure. The explicit expression for $G_{0}(0)$ [see Eq. (A8)] reads

$$
G_{0}(0)=\int \frac{d^{2} \mathbf{p}}{(2 \pi)^{2}} g_{1}(|\mathbf{p}|)[E+\sigma \cdot \mathbf{p}] .
$$

Evaluating $G_{0}(\mathbf{r})$ at the origin and setting $E=k$ yields

$$
G_{0}(0) \sim \int d p \frac{p}{k^{2}-p^{2}+i 0^{+}},
$$

which is logarithmic divergent. To obtain a physical meaningful result, a momentum cutoff, $p_{\max }$, in the upper limit of the integral must be considered. (This procedure is justified because graphene, being a solid, has an intrinsic energy cutoff of the order of the bandwidth.) We thus have

$$
G_{0}(0)=\frac{k}{2 \pi} \int_{0}^{p_{\max }} d p \frac{p}{k^{2}-p^{2}+i 0^{+}} .
$$

This integral yields

$$
G_{0}(0) \cong \frac{k}{2 \pi} \ln (k R),
$$

where we have assumed $k \ll p_{\max }$ and $R \equiv 1 / p_{\max }$ is a length scale of the order of $a_{0}$. Setting $\mathbf{r}=0$ in Eq. (A18), using the latter result and solving for $\Psi_{\mathbf{k}}(0)$ gives

$$
\begin{aligned}
\Psi_{\mathbf{k}}(0) & =\left[1-\frac{V_{0}}{2 \pi} k \ln (k R)\right]^{-1} \phi_{\mathbf{k}}(0) \\
& =\left[1-\frac{V_{0}}{2 \pi} k \ln (k R)\right]^{-1} u_{\mathbf{k}}^{(1)} .
\end{aligned}
$$

To identify the scattered amplitude, we need the asymptotic form of the Lippmann-Schwinger equation [Eq. (A4)]. For short-range potentials the main contribution in Eq. (A4) comes from the region where $\left|\mathbf{r}-\mathbf{r}^{\prime}\right| \gg 1$. Inserting the exact form of the propagator in space representation [Eq. (A12)] and using

$$
\begin{gathered}
H_{0}^{(1)}\left(k\left|\mathbf{r}-\mathbf{r}^{\prime}\right|\right) \rightarrow \sqrt{\frac{2}{i \pi k\left|\mathbf{r}-\mathbf{r}^{\prime}\right|}} e^{i k\left|\mathbf{r}-\mathbf{r}^{\prime}\right|}, \\
H_{1}^{(1)}\left(k\left|\mathbf{r}-\mathbf{r}^{\prime}\right|\right) \rightarrow-i \sqrt{\frac{2}{i \pi k\left|\mathbf{r}-\mathbf{r}^{\prime}\right|}} e^{i k\left|\mathbf{r}-\mathbf{r}^{\prime}\right|}
\end{gathered}
$$

leads to

$$
\begin{aligned}
\Psi_{\mathbf{k}}(\mathbf{r})= & \phi_{\mathbf{k}}(\mathbf{r})-\sqrt{\frac{i k}{8 \pi r}} e^{i k r} \\
& \times \int d^{2} \mathbf{r}^{\prime} e^{-i \mathbf{k}_{\mathrm{out}} \cdot \mathbf{r}^{\prime}}\left(1+\sigma_{\theta}\right) V_{d}\left(\mathbf{r}^{\prime}\right) \Psi_{\mathbf{k}}\left(\mathbf{r}^{\prime}\right),
\end{aligned}
$$

where we have approximated $\left|\mathbf{r}-\mathbf{r}^{\prime}\right| \simeq \mathbf{r}-\mathbf{r} \cdot \mathbf{r}^{\prime} / r$ and identified the wave vector at the point of observation, $\mathbf{k}_{\text {out }} \equiv k \mathbf{r} / r$. The exact form of the spinor at the origin [Eq. (A23)] allows us to find the explicit expression of $\Psi_{\mathbf{k}}(\mathbf{r})$; letting $\tilde{\sigma}_{\theta} \equiv \sigma_{\theta}\left(\mathbf{r}^{\prime}=0\right)$,

$$
\begin{aligned}
\Psi_{\mathbf{k}}(\mathbf{r})= & \phi_{\mathbf{k}}(\mathbf{r})-\frac{V_{0}}{1-\frac{V_{0}}{2 \pi} k \ln (k R)} \\
& \times \sqrt{\frac{i k}{8 \pi r}} e^{i k r}\left(1+\tilde{\sigma}_{\theta}\right) u_{\mathbf{k}}^{(1)} .
\end{aligned}
$$

The action of $\left(1+\tilde{\sigma}_{\theta}\right)$ on the spinor $u_{\mathbf{k}}^{(1)}$ yields the Berry phase term for scattering in graphene; without loss of generality, we take the incident momentum along the $x$ axis, $\mathbf{k}=(k, 0)$, and thus

$$
\begin{aligned}
\left(1+\tilde{\sigma}_{\theta}\right) u_{\mathbf{k}}^{(1)} & =\Xi_{B}(\theta) \frac{1}{\sqrt{2} A}\left(\begin{array}{c}
1 \\
e^{i \theta}
\end{array}\right) \\
& \equiv \Xi_{B}(\theta) u_{\mathbf{k}_{\text {out }}}^{(1)},
\end{aligned}
$$

where

$$
\Xi_{B}(\theta)=\left(1+e^{-i \theta}\right),
$$

and the scattering angle reads $\theta=\angle\left(\mathbf{k}, \mathbf{k}_{\text {out }}\right)$ [recall Eq. (A11) and comments therein].

The wave function of the scattered particles is then

$$
\Psi_{\mathbf{k}}(\mathbf{r})=\phi_{\mathbf{k}}(\mathbf{r})+f(\theta) \frac{e^{i k r}}{\sqrt{r}} u_{\mathbf{k}_{\text {out }}}^{(1)},
$$

with the scattering amplitude reading,

$$
f(\theta)=-\frac{1}{\hbar v_{F}} \sqrt{\frac{i k}{8 \pi}} \frac{V_{0}}{1-\frac{V_{0}}{2 \pi \hbar v_{F}} k \ln (k R)} \Xi_{B}(\theta),
$$

where we have restored all the constants. (Note that here $V_{0}$ has units of [energy] $\times$ [length $]^{2}$; the relation between $V_{0}$ and the effective impurity potential $V_{\text {eff }}$ in a lattice theory can be shown to be $V_{0} \sim A_{c} V_{\text {eff }}$, where $A_{c}$ is the area of graphene's unit cell.) This result is to be compared with the result from the FBA, which amounts to approximate $\Psi_{\mathbf{k}}\left(\mathbf{r}^{\prime}\right)$ by the unperturbed wave function $\phi_{\mathbf{k}}\left(\mathbf{r}^{\prime}\right)$ in Eq. (A26):

$$
f_{\text {Born }}(\theta)=-\frac{1}{\hbar v_{F}} \sqrt{\frac{i k}{8 \pi}} V_{0} \Xi_{B}(\theta) .
$$

The latter is only accurate in the limit of a very small $V_{0}$, which is of limited interest. The nonperturbative result discloses a singular momentum, $k_{\text {sing }}$,

$$
k_{\text {sing }} \ln \left(k_{\text {sing }} R\right)=\frac{2 \pi \hbar v_{F}}{V_{0}},
$$

which corresponds to a bound state of our problem. More importantly, the nonperturbative amplitude for $V_{0} \rightarrow \infty$ [recall that resonant scatterers in graphene give origin to strong 
short-range potentials; see Sec. (III A)] reads

$$
f_{V \rightarrow \infty}(\theta)=\sqrt{\frac{i \pi}{2}} \frac{\Xi_{B}(\theta)}{\sqrt{k} \ln (k R)},
$$

which is the main result of the present section.

\section{Nonperturbative amplitude for bilayer graphene}

Calculation of the scattering amplitude for the bilayer graphene follows as in Sec. A1, albeit with the important difference that the Green function does not diverge at the origin and thus no renormalization procedure is needed this time. This explains why no regularization length appears in the final result for the conductivity of bilayer graphene. We now outline the derivation of this result.

The explicit expression for $G_{0}(0)$ [see Eq. (A15)] reads

$$
G_{0}(0)=\int \frac{d^{2} \mathbf{p}}{(2 \pi)^{2}} g_{2}(\mathbf{p})[E+\gamma \boldsymbol{\sigma} \cdot \tilde{\mathbf{D}}(\mathbf{p})],
$$

which, setting $E=\gamma k^{2}$, can be simplified to

$$
G_{0}(0)=\gamma k^{2} \int \frac{d^{2} \mathbf{p}}{(2 \pi)^{2}} \frac{1}{\left(\gamma k^{2}+i 0^{+}\right)^{2}-\gamma^{2} p^{4}} .
$$

The above integral can be solved straightforwardly by contour integration; the result is

$$
G_{0}(0)=-\frac{i}{8 \gamma} .
$$

The amplitude of the wave function at the origin [Eq. (A18)] therefore reads

$$
\Psi_{\mathbf{k}}(0)=\left[1+i \frac{V_{0}}{8 \gamma}\right]^{-1} \phi_{\mathbf{k}}(0) .
$$

To identify the scattered amplitude, we have to repeat the derivation of the asymptotic form of the Lippmann-Schwinger equation [see Eqs. (A24)-(A40)]. The asymptotic form of the propagator can be calculated from Eq. (A17),

$$
\begin{aligned}
G_{0}\left(\mathbf{r}-\mathbf{r}^{\prime}\right) \rightarrow & -\frac{1}{4 \gamma} \sqrt{\frac{i}{2 k \pi\left|\mathbf{r}-\mathbf{r}^{\prime}\right|}} \\
& \times\left(\begin{array}{cc}
1 & e^{-2 i \theta\left(\mathbf{r}, \mathbf{r}^{\prime}\right)} \\
e^{2 i \theta\left(\mathbf{r}, \mathbf{r}^{\prime}\right)} & 1
\end{array}\right) e^{i k\left|\mathbf{r}-\mathbf{r}^{\prime}\right|},
\end{aligned}
$$

where $\theta=\angle\left(\mathbf{r}, \mathbf{r}^{\prime}\right)$. Inserting the latter expression into Eq. (A4), and approximating $\left|\mathbf{r}-\mathbf{r}^{\prime}\right| \simeq \mathbf{r}-\mathbf{r} \cdot \mathbf{r}^{\prime} / r$, permits us to identify the wave vector at the point of observation, $\mathbf{k}_{\text {out }} \equiv k \mathbf{r} / r$,

$$
\begin{aligned}
\Psi_{\mathbf{k}}(\mathbf{r})= & \phi_{\mathbf{k}}(\mathbf{r})-\frac{1}{4 \gamma} \sqrt{\frac{i}{2 k \pi r}} e^{i k r} \int d^{2} \mathbf{r}^{\prime} e^{-i \mathbf{k}_{\text {out }} \cdot \mathbf{r}^{\prime}} \\
& \times\left(1+\sigma_{2 \theta\left(\mathbf{r}, \mathbf{r}^{\prime}\right)}\right) V_{d}\left(\mathbf{r}^{\prime}\right) \Psi_{\mathbf{k}}\left(\mathbf{r}^{\prime}\right),
\end{aligned}
$$

where the definition of $\sigma_{\theta}$ is given in Eq. (A11). As before, letting $\tilde{\sigma}_{2 \theta} \equiv \sigma_{\theta\left(\mathbf{r}, \mathbf{r}^{\prime}=0\right)}$ and using Eq. (A38), we get

$$
\begin{aligned}
\Psi_{\mathbf{k}}(\mathbf{r})= & \phi_{\mathbf{k}}(\mathbf{r}) \\
& -\frac{2 V_{0}}{8 \gamma+i V_{0}} \sqrt{\frac{i}{2 k \pi r}} e^{i k r}\left(1+\tilde{\sigma}_{2 \theta}\right) u_{\mathbf{k}}^{(2)} .
\end{aligned}
$$

The action of the last term on the spinor $u_{\mathbf{k}}^{(2)}$ yields the bilayer Berry phase term; taking the incident momentum along the $x$ axis, $\mathbf{k}=(k, 0)$, we obtain

$$
\begin{aligned}
\left(1+\tilde{\sigma}_{2 \theta}\right) u_{\mathbf{k}}^{(2)} & =\Xi_{B}(2 \theta) \frac{1}{\sqrt{2} A}\left(\begin{array}{c}
1 \\
e^{2 i \theta}
\end{array}\right) \\
& \equiv \Xi_{B}(2 \theta) u_{\mathbf{k}_{\text {out }}^{(2)}}^{(2)}
\end{aligned}
$$

where $\Xi_{B}$ is defined in Eq. (A29) and $\theta$ is the scattering angle, $\theta=\angle\left(\mathbf{k}, \mathbf{k}_{\text {out }}\right)$ [recall Eq. (A11) and comments about it]. The wave function of the scattered particles is then

$$
\Psi_{\mathbf{k}}(\mathbf{r})=\phi_{\mathbf{k}}(\mathbf{r})+f(\theta) \frac{e^{i k r}}{\sqrt{r}} u_{\mathbf{k}_{\text {out }}}^{(2)},
$$

with the scattering amplitude reading

$$
f(\theta)=-\sqrt{\frac{i}{2 k \pi}} \frac{2 V_{0}}{8 v_{F}^{2} \hbar^{2} / t_{\perp}+i V_{0}} \Xi_{B}(2 \theta),
$$

where we have restored all the constants. The FBA is recovered in the limit $V_{0} \ll$ energy scales,

$$
f_{\mathrm{Born}}(\theta)=-\frac{V_{0}}{4 v_{F}^{2} \hbar^{2} / t_{\perp}} \sqrt{\frac{i}{2 k \pi}} \Xi_{B}(2 \theta) .
$$

In contrast, in the limit of interest $V_{0} \rightarrow \infty$, we obtain

$$
f_{V \rightarrow \infty}(\theta)=-\sqrt{\frac{2}{i \pi}} \frac{\Xi_{B}(2 \theta)}{\sqrt{k}} .
$$

\section{The de conductivity of monolayer and bilayer graphene}

The dc conductivity follows from the Boltzmann equation (see Sec. III B). The expression for the semiclassical current $\boldsymbol{j}$ can be manipulated to yield a more convenient form of the conductivity for our purposes. We reproduce the main steps; at $T=0$ the Fermi function becomes the Heaviside function, $\theta\left(\epsilon_{k_{F}}-\epsilon_{k}\right)$, and hence the expression for the current reads

$$
\boldsymbol{j}=\frac{g_{s} g_{v} e^{2}}{(2 \pi)^{2}} \int d \mathbf{k} \tau(k) \delta\left(\epsilon_{k_{F}}-\epsilon_{k}\right)\left(\mathbf{v}_{k} \cdot \mathbf{E}\right) \mathbf{v}_{k} .
$$

Performing the angular integration, and using the relation $v_{r}=$ $\hbar^{-1} \partial_{k} \epsilon$, leads to

$$
\boldsymbol{j}=\frac{e^{2}}{\pi \hbar} \frac{k_{F}}{\left|v_{r}\left(k_{F}\right)\right|} \tau\left(k_{F}\right)\left(\mathbf{v}_{k_{F}} \cdot \mathbf{E}\right) \mathbf{v}_{k_{F}} .
$$

The longitudinal dc conductivity follows from the latter expression:

$$
\sigma_{\mathrm{dc}}=\frac{2 e^{2}}{h} \tau\left(k_{F}\right)\left|v_{r}\left(k_{F}\right)\right| k_{F} .
$$

Using the results in Secs. A1 and A2 and the definition of relaxation time $\tau\left(k_{F}\right)$ (Sec. III B), we can readily obtain the dc conductivity in the regime of $V_{0} \gg$ energy scales. (For a discussion of the on-site energy $V_{0}$ magnitude, see Sec. III A.)

The dc conductivity in the limit $V_{0} \rightarrow \infty$ reads

$$
\sigma_{\mathrm{dc}}^{\text {strong }}= \begin{cases}\frac{4 e^{2}}{h} \frac{k_{F}^{2}}{2 \pi^{2} n_{i}} \ln ^{2}\left(k_{F} R\right) & \text { for monolayer, } \\ \frac{4 e^{2}}{h} \frac{k_{F}^{2}}{16 n_{i}} & \text { for bilayer. }\end{cases}
$$

As expected, the dependence on $k_{F}$ coincides with that obtained through the partial-wave expansion method employed in 
Sec. III. Remarkably, the expressions match exactly [compare with Eqs. (63) and (71)]. This entails that scattering off a hard disk of radius $R \sim a_{0}$ and scattering off a strong $\delta$ potential have the same dependence on the momentum of the incident particles (in both monolayer and bilayer graphene).
*Corresponding author: peres@ fisica.uminho.pt

${ }^{1}$ R. Peierls, Surprises in Theoretical Physics (Princeton University Press, Princeton, NJ, 1979), p. 1.

${ }^{2}$ K. S. Novoselov, A. K. Geim, S. V. Morozov, D. Jiang, Y. Zhang, S. V. Dubonos, I. V. Grigorieva, and A. A. Firsov, Science 306, 666 (2004).

${ }^{3}$ K. S. Novoselov, D. Jiang, T. Booth, V. V. Khotkevich, S. M. Morozov, and A. K. Geim, Proc. Natl. Acad. Sci. USA 102, 10451 (2005).

${ }^{4}$ A. H. Castro Neto, F. Guinea, N. M. R. Peres, K. S. Novoselov, and A. K. Geim, Rev. Mod. Phys. 81, 109 (2009).

${ }^{5}$ N. M. R. Peres, Rev. Mod. Phys. 82, 2673 (2010).

${ }^{6}$ D. K. Efetov and P. Kim, e-print arXiv:1009.2988 (2010).

${ }^{7}$ W. A. de Heer et al., Solid State Commun. 143, 92 (2007).

${ }^{8}$ X. Li, W. Cai, J. An, S. Kim, J. Nah, D. Yang, R. Piner, A. Velamakanni, I. Jung, E. Tutuc, S. K. Banerjee, L. Colombo, and R. S. Ruoff, Science 324, 1312 (2009).

${ }^{9}$ K. S. Kim, Y. Zhao, H. Jang, S. Y. Lee, J. M. Kim, K. S. Kim, J.-H. Ahn, P. Kim, J.-Y. Choi, and B. H. Hong, Nature 457, 706 (2009).

${ }^{10}$ A. Ferreira et al., e-print arXiv:1008.0618 (2010).

${ }^{11}$ V. M. Pereira, A. H. Castro Neto, and N. M. R. Peres, Phys. Rev. B 80, 045401 (2009).

${ }^{12}$ J. C. Meyer, A. K. Geim, M. I. Katsnelson, K. S. Novoselov, T. J. Booth, and S. Roth, Nature 446, 60 (2007).

${ }^{13}$ M. I. Katsnelson and A. K. Geim, Philos. Trans. R. Soc. London A 366, 195 (2008).

${ }^{14}$ M. M. Fogler, A. H. Castro Neto, and F. Guinea, Phys. Rev. B 81, 161408(R) (2010).

${ }^{15}$ Y. Zhang, V. W. Brar, C. Girit, A. Zettl, and M. F. Crommie, Nature Phys. 5, 722 (2009).

${ }^{16}$ S. Adam, E. H. Hwang, and S. Das Sarma, Physica E 40, 1022 (2008).

${ }^{17}$ S. Adam, E. H. Hwang, V. Galitski, and S. Das Sarma, Proc. Natl. Acad. Sci. USA 104, 18392 (2007).

${ }^{18}$ E. H. Hwang and S. Das Sarma, Phys. Rev. B 77, 195412 (2008).

${ }^{19}$ J. Martin, N. Akerman, G. Ulbricht, T. Lohmann, J. H. Smet, K. von Klitzing, and A. Yacoby, Nature Phys. 4, 144 (2008).

${ }^{20}$ T. O. Wehling, S. Yuan, A. I. Lichtenstein, A. K. Geim, and M. I. Katsnelson, Phys. Rev. Lett. 105, 056802 (2010).

${ }^{21}$ J.-H. Chen, W. G. Cullen, C. Jang, M. S. Fuhrer, and E. D. Williams, Phys. Rev. Lett. 102, 236805 (2009).

${ }^{22}$ M. Monteverde, C. Ojeda Aristizabal, R. Weil, K. Bennaceur, M. Ferrier, S. Gueron, C. Glattli, H. Bouchiat, J. N. Fuchs, and D. L. Maslov, Phys. Rev. Lett. 104, 126801 (2010).

${ }^{23}$ Z. H. Ni, L. A. Ponomarenko, R. R. Nair, R. Yang, S. Anissimova, I. V. Grigorieva, F. Schedin, Z. X. Shen, E. H. Hill, K. S. Novoselov, and A. K. Geim, Nano Lett. (in press, 2010).

${ }^{24}$ J. Katoch, J.-H. Chen, R. Tsuchikawa, C. W. Smith, E. R. Mucciolo, and M. Ishigami, Phys. Rev. B 82, 081417(R) (2010).

${ }^{25}$ N. M. R. Peres, F. Guinea, and A. H. Castro Neto, Phys. Rev. B 73, 125411 (2006).
${ }^{26}$ V. M. Pereira, F. Guinea, J. M. B. Lopes dos Santos, N. M. R. Peres, and A. H. Castro Neto, Phys. Rev. Lett. 96, 036801 (2006).

${ }^{27}$ V. M. Pereira, J. M. B. Lopes dos Santos, and A. H. Castro Neto, Phys. Rev. B 77, 115109 (2008).

${ }^{28}$ C. H. Yang, F. M. Peeters, and W. Xu, Phys. Rev. B 82, 205428 (2010).

${ }^{29}$ D. S. Novikov, Phys. Rev. B 76, 245435 (2007).

${ }^{30}$ M. I. Katsnelson, Phys. Rev. B 76, 073411 (2007).

${ }^{31}$ D. M. Basko, Phys. Rev. B 78, 115432 (2008).

${ }^{32}$ M. Hentschel and F. Guinea, Phys. Rev. B 76, 115407 (2007).

${ }^{33}$ S. K. Adhikari, Am. J. Phys. 54, 362 (1986).

${ }^{34}$ L. L. Schif, Quantum Mechanics, 3rd ed. (McGraw-Hill, New York, 1968).

${ }^{35} \mathrm{M}$. Abramowitz and I. A. Stegun, Handbook of Mathematical Functions (Dover, Mineola, NY, 1965).

${ }^{36}$ E. V. Castro, K. S. Novoselov, S. V. Morozov, N. M. R. Peres, J. Lopes dos Santos, J. Nilsson, F. Guinea, A. K. Geim, and A. H. Castro Neto, Phys. Rev. Lett. 99, 216802 (2007).

${ }^{37}$ E. V. Castro, K. S. Novoselov, S. V. Morozov, N. M. R. Peres, J. Lopes dos Santos, J. Nilsson, F. Guinea, A. K. Geim, and A. H. Castro Neto, J. Phys. Condens. Matter 22, 175503 (2010).

${ }^{38}$ E. McCann and V. I. Falko, Phys. Rev. Lett. 96, 086805 (2006).

${ }^{39}$ E. McCann, Phys. Rev. B 74, 161403(R) (2006).

${ }^{40}$ M. I. Katsnelson, K. S. Novoselov, and A. K. Geim, Nature Phys. 2, 620 (2006).

${ }^{41}$ J. P. Robinson, H. Schomerus, L. Oroszlany, and V. I. Fal'ko, Phys. Rev. Lett. 101, 196803 (2008).

${ }^{42}$ T. Stauber, N. M. R. Peres, and F. Guinea, Phys. Rev. B 76, 205423 (2007).

${ }^{43}$ T. O. Wehling, M. I. Katsnelson, and A. I. Lichtenstein, Phys. Rev. B 80, 085428 (2009).

${ }^{44}$ E. V. Castro, M. P. López-Sancho, and M. A. H. Vozmediano, Phys. Rev. Lett. 104, 036802 (2010).

${ }^{45}$ N. M. R. Peres, L. Yang, and S.-W. Tsai, New J. Phys. 11, 095007 (2009).

${ }^{46}$ N. M. R. Peres, F. D. Klironomos, S.-W. Tsai, J. R. Santos, J. M. B. L. dos Santos, and A. H. C. Neto, Europhys. Lett. 80, 67007 (2007).

${ }^{47}$ C. Bena and S. A. Kivelson, Phys. Rev. B 72, 125432 (2005).

${ }^{48}$ J. M. Ziman, Principles of the Theory of Solids, 2nd ed. (Cambridge University Press, Cambridge, 1979).

${ }^{49}$ F. Dominguez-Adamé, J. Phys. A Math. Gen. 23, 1993 (1990).

${ }^{50}$ S. V. Morozov, K. S. Novoselov, M. I. Katsnelson, F. Schedin, D. C. Elias, J. A. Jaszczak, and A. K. Geim, Phys. Rev. Lett. 100, 016602 (2008).

${ }^{51}$ P. M. Ostrovsky, I. V. Gornyi, and A. D. Mirlin, Phys. Rev. B 74, 235443 (2006)

${ }^{52}$ B. Huard, N. Stander, J. A. Sulpizio, and D. Goldhaber-Gordon, Phys. Rev. B 78, 121402R (2008).

${ }^{53}$ K. Nomura and A. H. MacDonald, Phys. Rev. Lett. 98, 076602 (2007). 
${ }^{54}$ J. Nilsson, A. H. Castro Neto, F. Guinea, and N. M. R. Peres, Phys. Rev. Lett. 97, 266801 (2006).

${ }^{55}$ J. Nilsson, A. H. Castro Neto, F. Guinea, and N. M. R. Peres, Phys. Rev. B 78, 045405 (2008).

${ }^{56}$ M. Koshino, New J. Phys. 11, 095010 (2009).

${ }^{57}$ S. Das Sarma, E. H. Hwang, and E. Rossi, Phys. Rev. B 81, 161407 (2010).

${ }^{58}$ S. Adam and S. Das Sarma, Phys. Rev. B 77, 115436 (2008).

${ }^{59}$ J. W. Kłos and I. V. Zozoulenko, Phys. Rev. B 82, 081414(R) (2010).

${ }^{60}$ E. R. Mucciolo and C. H. Lewenkopf, J. Phys. Condens. Matter 22, 273201 (2010).

${ }^{61}$ G. D. Mahan, Many-Particle Physics, 3rd ed. (Springer, New York, 2000).

${ }^{62}$ A. Ferreira, E. R. Mucciolo, and N. M. R. Peres, in preparation.

${ }^{63}$ A. Weiße, G. Wellein, A. Alvermann, and H. Fehske, Rev. Mod. Phys 78, 275 (2006).

${ }^{64}$ P. M. Ostrovsky, M. Titov, S. Bera, I. V. Gornyi, and A. D. Mirlin, e-print arXiv:1006.3299 (2010).

${ }^{65}$ M. Titov, Europhys. Lett. 79, 17004 (2007).

${ }^{66}$ C. H. Lewenkopf, E. R. Mucciolo, and A. H. Castro Neto, Phys. Rev. B 77, 081410R (2008).
${ }^{67}$ F. Guinea, A. H. Castro Neto, and N. M. R. Peres, Phys. Rev. B 73, 245426 (2006).

${ }^{68}$ T. Stauber, N. M. R. Peres, F. Guinea, and A. H. Castro Neto, Phys. Rev. B 75, 115425 (2007).

${ }^{69}$ F. Xia, D. B. Farmer, Y. M. Lin, and P. Avouris, Nano Lett. 10, 715 (2010).

${ }^{70} \mathrm{~S}$. Weinberg, The Quantum Theory of Fields I (Cambridge University Press, Cambridge, 1995).

${ }^{71} \mathrm{~A}$ more accurate calculation of the density of states starts from the spectrum as calculated directly from the four-band model, Eq. (26), and yields $\rho(E)=\left(t_{\perp}+2 E\right) /\left(\pi \sqrt{3} t^{2}\right)$. The equation given in the text is recovered for $E \ll t_{\perp}$.

${ }^{72}$ S. Yuan, Hans De Raedt, and M. I. Katsnelson, Phys. Rev. B 82, 115448 (2010)

${ }^{73}$ E. McCann, K. Kechedzhi, Vladimir I. Falõko, H. Suzuura, T. Ando, and B. L. Altshuler, Phys. Rev. Lett. 97, 146805 (2006).

${ }^{74}$ F. V. Tikhonenko, D. W. Horsell, R. V. Gorbachev, and A. K. Savchenko, Phys. Rev. Lett. 100, 056802 (2008).

${ }^{75}$ M. G. Menezes, R. B. Capaz, and J. L. B. Faria, Phys. Rev B 82, 245414 (2010)

${ }^{76}$ N. Leconte et al., ACS Nano 4, 4033 (2010).

${ }^{77}$ S. Yuan, H. De Raedt, and M. I. Katsnelson, e-print arXiv:1010.2858 (2010). 